\title{
POINCARÉ FUNCTION FOR MODULI OF DIFFERENTIAL-GEOMETRIC STRUCTURES
}

\author{
BORIS KRUGLIKOV
}

\begin{abstract}
The Poincaré function is a compact form of counting moduli in local geometric problems. We discuss its property in relation to V. Arnold's conjecture, and derive this conjecture in the case when the pseudogroup acts algebraically and transitively on the base. Then we survey the known counting results for differential invariants and derive new formulae for several other classification problems in geometry and analysis.
\end{abstract}

\section{INTRODUCTION}

The Poincaré function counts the number of moduli in algebrogeometric problems. Classically, for a graded algebra $A=\oplus_{i \geq 0} A_{i}$ with $a_{i}=\operatorname{dim} A_{i}<\infty$, this function is given by

$$
P(z)=\sum_{i=0}^{\infty} a_{i} z^{i} .
$$

In particular, this applies to the algebra of invariants $A=R[X]^{G}$ of an algebraic action of a Lie group $G$ on a variety $X$. It encodes grows of the number of invariants with their algebraic degree. For semi-simple Lie groups $G$ this has received numerous applications, see e.g. description of invariants and covariants of binary forms in [36].

In the same vein, the Poincaré function is used in the local analysis of differential-geometric problems. As the setup let $G$ be an algebraic pseudogroup (the definitions will be recalled in Section 1) acting on a space $\mathcal{E}$ of geometric objects, which can be the space of sections of a tensor bundle of prescribed type or the sheaf of solutions to a certain geometric equation. However instead of considering global sections or germs of those, we shall conveniently work with their jets.

Thus $\mathcal{E}$ consists of (jets of) sections of a bundle $\pi$ and $G$ consists of local diffeomorphisms of $J^{0} \pi$. Prolong the action of $G$ to higher jets $J^{k} \pi$, possibly restricting to invariant subbundles $\mathcal{E}^{k}$ such that the projections $\pi_{k, k-1}: \mathcal{E}^{k} \rightarrow \mathcal{E}^{k-1}$ are submersions. Thus $\mathcal{E}$ is a co-filtered

Key words and phrases. Differential Invariants, Invariant Derivations, conformal metric structure, Hilbert polynomial, Poincaré function. 
manifold, also known as diffiety, the projective limit of $\mathcal{E}^{k}$. In most cases of interest $\mathcal{E}$ is either un-constrained or a formally integrable differential equation. A more general setup will be given in Section 1 .

Due to algebraic nature of the action, it possesses a rational quotient $\mathcal{Q}_{k}=\mathcal{E}^{k} / G$ (space of $G$-orbits in $\mathcal{E}^{k}$ ) by the Rosenlicht theorem [32] and the prolongation result of [23]. Rational functions on $\mathcal{Q}_{k}$ are bijective with $G$-invariant rational functions on $\mathcal{E}^{k}$ and are called (global) scalar differential invariants of order $k$; their pole divisors are $G$-invariant.

Dimension $s_{k}$ of $\mathcal{Q}_{k}$ is equal to the transcendence degree of the field of rational differential invariants of order $\leq k$, and it corresponds to the number of (functionally) independent invariants of such order. The difference $h_{k}=s_{k}-s_{k-1}$ can be interpreted as the number of "pure order" $k$ differential invariants.

Under certain assumptions, the sequence $h_{k}$ (as well as $s_{k}$ ) or its arithmetic sub-sequence $h_{a k+b}$ (where $a \in \mathbb{N}$ is fixed, and $0 \leq b<a$ varies) is a polynomial for $k \gg 1$, called the Hilbert polynomial. This fact was experimentally observed by V. Arnold for some local problems in analysis and geometry [1]. Later this conjecture was proved for the diffeomorphism pseudogroups acting on natural geometric bundles in [33, and then for general algebraic pseudogroups acting on algebraic differential equations in [23]. The basic assumption in the last reference, in addition to algebraicity, is transitivity of the pseudogroup action on the base manifold, and we adapt this also in what follows.

The Poincaré function of this action is defined by

$$
P(z)=\sum_{k=0}^{\infty} h_{k} z^{k}
$$

The series clearly converges and gives an analytic function in the disk $|z|<1$. Under the above assumptions, it is a rational function with the only pole at $z=1$. The Hilbert function is restored by the formula

$$
h_{k}=\operatorname{Res}_{z=0} \frac{P(z)}{z^{k+1}}=\left.\frac{1}{k !} \frac{d^{k}}{d z^{k}}\right|_{z=0} P(z),
$$

and the number of independent differential invariants of order $\leq k$ by the formula (in both cases $k \gg 1$ )

$$
s_{k}=\operatorname{Res}_{z=-1} \frac{P(z+1)}{z}-\operatorname{Res}_{z=0} \frac{P(z)}{z^{k+1}(z-1)} .
$$

Thus Poincaré function is the generating function encoding the count for differential invariants.

Often, the quotient $\mathcal{Q}=\mathcal{E} / G$, co-filtered by $\mathcal{Q}_{k}$, has functional rank $\sigma$ and functional dimension $d$, meaning that the number of jets in $\mathcal{Q}$ is 
asymptotic to that for the space of jets of $\sigma$ functions of $d$ arguments: $s_{k} \sim \sigma \cdot\left(\begin{array}{c}k+d-1 \\ d-1\end{array}\right)$. This implies at once that

$$
P(z)=\frac{R(z)}{(1-z)^{d}},
$$

for some polynomial $R(z), R(1) \neq 0$, so the functional dimension $d$ is easily identifyable. The functional rank can be found by the change of variables $w=1-z: P(1-w)=\sigma w^{-d}+\sum_{i>-d} p_{i} w^{i}$ where the sum is finite. This yields $\sigma=R(1)$, so the functional dimension and rank express so:

$$
d=-\lim _{z \rightarrow 1} \frac{\log P(z)}{\log (1-z)}, \quad \sigma=\lim _{z \rightarrow 1} P(z)(1-z)^{d} .
$$

More complicated Poincaré series than (2), leading to different formulas for $(d, \sigma)$, are briefly discussed at the end of the paper, see Section 4 .

This paper has the following three goals:

(1) Discuss rationality of the function $P(z)$, deducing a strong form of Arnold's conjecture [1, Problem 1994-24] in the case the action is algebraic and transitive on the base, see Theorem 3 ,

(2) Provide explicit rational formulae for $P(z)$ in many classical examples, summarizing (sometimes correcting and generalizing) and compactifying the known results from the literature;

(3) Compute the Poincaré function for several new important cases, including an infinite type geometric structure, which is a novel local result in almost complex geometry.

These problems will be subsequently addressed in the further sections. Validity of (1) relies on a derivation of the main result in [23], which we recall in Section 1 and then indicate modifications required to achieve the claim. The tools important to compute the Poincaré function are given in Section 2, and then the results of (2) and (3) are presented in Section 3, constituting the main body of this paper. An outlook is given in Section 4 .

\section{On ARnOld's CONJECTURE}

A weak form of the Arnold conjecture states that the Poincaré function $P(z)$ is rational. The coefficient $h_{k}=s_{k}-s_{k-1}$ of $z^{k}$ in (1) expresses through the codimension $s_{k}$ of generic $G$-orbits in $\mathcal{E}^{k}$, but this $s_{k}$ can be also understood as codimension of a particular orbit $G \cdot a_{k}$ through $a_{k} \in \mathcal{E}^{k}$ and then it depends on this point. If the action is algebraic, then $s_{k}$ is constant on a Zariski open subset $\mathcal{E}_{k}^{\prime} \subset \mathcal{E}^{k}$ [32, 23]. Uniting these yields a Zariski open set $\mathcal{E}^{\prime} \subset \mathcal{E}$ on which $P(z)$ is rational [23]. 
A strong form of Arnold's conjecture states that there exists a subset $\Sigma \subset \mathcal{E}$ of codim $\Sigma=\infty$ and a (co-filtered) stratification $\mathcal{E} \backslash \Sigma=\cup_{\alpha} \mathcal{E}_{\alpha}$ such that with $s_{k}^{\alpha}=\operatorname{codim}\left(G \cdot a_{k} \subset \mathcal{E}^{k}\right), a_{k} \in \mathcal{E}_{\alpha}^{k}$, and $h_{k}^{\alpha}=s_{k}^{\alpha}-s_{k-1}^{\alpha}$, the corresponding Poincaré function $P_{\alpha}(z)=P\left(a_{\infty} ; z\right)=\sum_{k=0}^{\infty} h_{k}^{\alpha} z^{k}$ is rational for every $\alpha$ (i.e. for every $\left.a_{\infty}=\left\{a_{k}\right\}_{k=0}^{\infty} \in \mathcal{E}_{a}^{\infty}, a=a_{0} \in M\right)$.

1.1. A solution of the conjecture. A pseudogroup is a collection of local diffeomorphisms $G \subset \operatorname{Diff}_{\text {loc }}(M)$ that contains unit, inverse, and composition whenever defined. It is called a Lie pseudogroup if its elements are solutions to a system of differential equations, see [28, 34, 24]. Thus we identify $G$ with a projective limit of subsets $G^{k} \subset$ $J^{k}(M, M)$ that give a formally integrable Lie equation. Since local and formal diffeomorphisms have the same differential invariants (see below), we will not make a distinction between them.

Denote by $J_{n}^{k}(M)$ the space of $k$-jets of $n$-dimensional submanifolds $N \subset M$; note that $J^{k}(M, M) \subset J_{m}^{k}(M \times M)$ for $m=\operatorname{dim} M$. A differential equation $\mathcal{E}$ is a collection of submanifolds $\mathcal{E}^{k} \subset J_{n}^{k}, \mathcal{E}^{0}=$ $J_{n}^{0}=M$, such that the projections $\pi_{k, k-1}: \mathcal{E}^{k} \rightarrow \mathcal{E}^{k-1}$ are submersions (note the un-constraint case: $\mathcal{E}^{k}=J_{n}^{k}$ ). It is called formally integrable if $\mathcal{E}^{k}$ is a subset of the prolongation of $\mathcal{E}^{k-1}$, i.e. the defining relations of $\mathcal{E}^{k}$ are obtained by differentiations of those of $\mathcal{E}^{k-1}$. Recall that there is a natural algebraic structure on fibers of $J_{n}^{k}$. If the defining relations of $\mathcal{E}$ are algebraic (in jets of order $\geq 1$; for simplicity, we assume no relation of order zero is imposed on $\mathcal{E}$ ), the equation is called algebraic.

In particular, if the Lie equation is algebraic we call the pseudogroup $G$ algebraic. It naturally acts on the jet-spaces $J_{n}^{k}$, and the equation $\mathcal{E}$ is called $G$-invariant if $G \cdot \mathcal{E}^{k} \subset \mathcal{E}^{k}$. Equivalently, if $\mathcal{G}$ is the Lie algebra sheaf of $G$ (local vector fields $X=\left.\frac{d g_{t}}{d t}\right|_{t=0}$ for paths $g_{t} \subset G, g_{0}=\mathrm{Id}$ ), then $\mathcal{E}$ is $G$-invariant if $X_{a_{k}} \in T_{a_{k}} \mathcal{E}^{k}$ for all $X \in \mathcal{G}, a_{k} \in \mathcal{E}^{k}$.

A function $f$ on $\mathcal{E}$ is by definition a function $f: \mathcal{E}^{k} \rightarrow \mathbb{R}$ for some $k$ pulled back to $\mathcal{E}^{\infty}$. It is $G$-invariant if $g^{*} f=f$ for any $g \in G$. Provided $G$ is connected, this is equivalent to $L_{X} f=0$ for any $X \in \mathcal{G}$.

Consider the field of rational functions $\mathfrak{R}(\mathcal{E})=\cup_{k} \mathfrak{R}\left(\mathcal{E}^{k}\right)$ and its subfield of rational invariants $\mathfrak{F}=\mathfrak{R}(\mathcal{E})^{G}$. If $G$ is Zariski-connected (we assume this in what follows), $f$ is a rational differential invariant iff $L_{X} f=0$ for any $X \in \mathcal{G}$. By Rosenlicht's theorem [32], elements of $\mathfrak{F}_{k}=\mathfrak{R}\left(\mathcal{E}^{k}\right)^{G}$ separate regular $G$-orbits and the transcendence degree of $\mathfrak{F}_{k}$ is the codimension $s_{k}$ of a generic orbit in $\mathcal{E}^{k}$.

Remark. By [23] there exists a natural number l such that the subalgebra $\mathfrak{A}$ of invariant functions that are rational by jets of order $\leq l$ and polynomial by jets of higher order suffices to separate regular orbits. 
In addition to differential invariants one defines invariant derivations as first order operators in total derivatives $\nabla: \mathfrak{R}(\mathcal{E}) \rightarrow \mathfrak{R}(\mathcal{E})$ commuting with the action of $G$. Global Lie-Tresse theorem [23] states that the field $\mathfrak{F}$ (and the algebra $\mathfrak{A}$ ) is generated by a finite number of differential invariants $I_{i}$ and a finite number of invariant derivations $\nabla_{j}$. Moreover, loc.cit. proves that the invariant syzygies and higher syzygies are also finitely generated in the Lie-Tresse sense. This implies that $s_{k}$ and hence $h_{k}$ are polynomials in $k$ for $k \gg 1$, whence the following claim [23, Theorem 26]:

Theorem 1. Consider an algebraic action of a connected pseudogroup $G$ on an irreducible algebraic differential equation $\mathcal{E} \subset J_{n}^{\infty}(M)$. Assume that $G$ acts transitively on $M$. Then the Poincaré function $P(z)$ of this action is rational and has form (2), where the degree $d$ of the only pole $z=1$ does not exceed the degree of the complex affine characteristic variety of $\mathcal{E}$; in particular $d \leq n$.

This gives a solution of (the weak form of) Arnold's conjecture under the assumptions of the theorem, of which the most crucial is the transitivity of $G$-action on the base $M$. We will comment at the end of the paper on what happens when this assumption is violated.

1.2. A generalization: strong version of the conjecture. We claim that the previous statement holds true for a more general class of submanifolds $\mathcal{E}^{\infty} \subset J_{n}^{\infty}$ co-filtered by $\mathcal{E}^{k} \subset J_{n}^{k}$ as long as the basic assumptions of Theorem 1 are satisfied.

The setup is as follows. Let $\overline{\mathcal{E}}$ be a formally integrable differential equation co-filtered by $\overline{\mathcal{E}}^{k} \subset J_{n}^{k}$. Consider a finite number of functions (nonlinear differential operators) $\Phi_{s}: J_{n}^{k_{s}} \rightarrow \mathbb{R}$. Let $\sigma_{k}=\left\{s: k_{s} \leq k\right\}$. Define $\mathcal{E}^{k}=\left\{a_{k} \in \overline{\mathcal{E}}^{k}: \Phi_{s}\left(a_{s}\right)=0 \forall s \in \sigma_{k}\right\}$. We assume regularity: the projections $\pi_{k, k-1}: \mathcal{E}^{k} \rightarrow \mathcal{E}^{k-1}$ are submersions.

Thus we allow $\mathcal{E}^{k}$ to be not a part of the prolongation of $\mathcal{E}^{k-1}$, but this can happen only for a finite set of orders $k$. We call such $\mathcal{E}$ a generalized equation. In particular, we can start with $\overline{\mathcal{E}}^{\infty}=J_{n}^{\infty}$ and impose a finite number of differential equations $\left\{\Phi_{s}=0\right\}$ without including prolongations of those. If $\Phi_{s}$ are algebraic functions and $\overline{\mathcal{E}}$ is algebraic, we call the generalized equation $\mathcal{E}$ algebraic. $G$-invariance extends straightforwardly.

Consider a sequence of points $a_{k} \in \mathcal{E}^{k}, \pi_{k, k-1}\left(a_{k}\right)=a_{k-1}$, and let $a_{\infty}=\lim a_{k} \in \mathcal{E}^{\infty}$. If $a_{1}=[N]_{a}^{1}$ for a $n$-manifold $N \subset M$ then we denote $\tau_{a}=T_{a} N$ and $\nu_{a}=T_{a} M / T_{a} N$; they depend only on $a_{1}$. As for usual differential equations $g_{k}\left(a_{k}\right)=\operatorname{Ker}\left(d \pi_{k, k-1}: T_{a_{k}} \mathcal{E}^{k} \rightarrow T_{a_{k-1}} \mathcal{E}^{k-1}\right)$ is called the $k$-symbol of $\mathcal{E}$, and it is naturally identified with a subspace 
in $S^{k} \tau_{a}^{*} \otimes \nu_{a}=\operatorname{Ker}\left(d \pi_{k, k-1}: T_{a_{k}} J_{n}^{k} \rightarrow T_{a_{k-1}} J_{n}^{k-1}\right)$. Uniting these we get the symbolic system $g\left(a_{\infty}\right)=\left\{g_{k}\left(a_{k}\right)\right\} \subset S \tau_{a}^{*} \otimes \nu_{a}$.

Let $\delta: S^{i} \tau_{a}^{*} \otimes \nu_{a} \otimes \Lambda^{j} \tau^{*} \rightarrow S^{i-1} \tau_{a}^{*} \otimes \nu_{a} \otimes \Lambda^{j+1} \tau^{*}$ be the Spencer $\delta$ differential (symbol of the de Rham operator). When $\mathcal{E}$ is a differential equation and $g$ its symbol, the sequence

$$
\cdots \rightarrow g_{i+1} \otimes \Lambda^{j-1} \tau^{*} \stackrel{\delta}{\longrightarrow} g_{i} \otimes \Lambda^{j} \tau^{*} \stackrel{\delta}{\longrightarrow} g_{i-1} \otimes \Lambda^{j+1} \tau^{*} \stackrel{\delta}{\rightarrow} \cdots
$$

is the Spencer complex; its cohomology at the $(i, j)$-term $H^{i, j}\left(\mathcal{E} ; a_{\infty}\right)=$ $H^{i, j}(g)$ is called the Spencer $\delta$-cohomology group.

For a generalized equation $\mathcal{E}$ the map $\delta$ on $g_{i} \otimes \Lambda^{j} \tau^{*}$ may not take values in $g_{i-1} \otimes \Lambda^{j+1} \tau^{*}$ when $i$ is an order, i.e. $\sigma_{i} \neq \sigma_{i-1}$. However for $i$ exceeding the maximum order the $\delta$-differential is well-defined, so if, in addition, $i$ exceeds the involutivity order of $\overline{\mathcal{E}}$ then $H^{i, *}(g)=0$.

Theorem 2. Consider an algebraic action of a pseudogroup $G$ on an algebraic generalized differential equation $\mathcal{E} \subset J_{n}^{\infty}(M)$. Let $G$ act transitively on $M$. Then the Poincaré function $P(z)$ of this action is rational of the form (2). It has only one pole at $z=1$ of degree $d \leq n$. Moreover, $P(z)=\stackrel{P}{P}\left(a_{\infty} ; z\right)$ is locally constant by $a_{\infty}$ when this point vary in a component of a Zariski open set $\mathcal{E}^{\prime \prime} \subset \mathcal{E}$.

Proof. Note that we allow $\mathcal{E}$ to be reducible. In this case we restrict to one of its finitely many components. Thus the claim follows from an irreducible case, on which we now concentrate.

Let $\Delta_{k}\left(a_{k}\right)=T_{a_{k}}\left(G^{k} \cdot a_{k}\right)=\left\{X_{a_{k}}^{(k)}: X \in \mathcal{G}\right\}$ be the tangent differential system. For a point $a_{\infty} \in \mathcal{E}^{\infty}$ consider the subspace

$$
\varpi_{k}=\operatorname{Ker}\left(d \pi_{k, k-1}: \Delta_{k}\left(a_{k}\right) \rightarrow \Delta_{k-1}\left(a_{k-1}\right)\right) \subset S^{k} \tau_{a}^{*} \otimes \nu_{a} .
$$

The main observation is that the proofs of Proposition 10 and Theorem 11 of [23] use only the surjectivity of $\pi_{k, k-1}: \mathcal{E}^{k} \rightarrow \mathcal{E}^{k-1}$ and algebraicity of the action. Thus we we can apply Corollary 12 of loc.cit. to conclude that there exists a natural $l$ and a Zariski open subset $\mathcal{E}^{\prime \prime}=\pi_{\infty, l}^{-1}\left(\mathcal{E}_{l}^{\prime \prime}\right) \subset \mathcal{E}$ such that for all $i \geq l, j \geq 0$ and $a_{\infty} \in \mathcal{E}^{\prime \prime}$ the sequence

$$
\cdots \rightarrow \varpi_{i+1} \otimes \Lambda^{j-1} \tau^{*} \stackrel{\delta}{\longrightarrow} \varpi_{i} \otimes \Lambda^{j} \tau^{*} \stackrel{\delta}{\longrightarrow} \varpi_{i-1} \otimes \Lambda^{j+1} \tau^{*} \stackrel{\delta}{\rightarrow} \cdots
$$

is well-defined and is exact: $H^{i, j}(\varpi)=0$.

Denote $\mathfrak{d}_{k}=\operatorname{Ker}\left(T \mathcal{Q}_{k} \rightarrow T \mathcal{Q}_{k-1}\right)$, where $\mathcal{Q}_{k}=\mathcal{E}^{k} / G$ is the rational quotient. Then the exact sequences

$$
0 \rightarrow \varpi_{k} \longrightarrow g_{k} \longrightarrow \mathfrak{d}_{k} \rightarrow 0
$$

and the corresponding Spencer $\delta$-complexes unite into a bi-complex, which by the snake lemma implies that $H^{i, j+1}(\varpi)=H^{i+1, j}(\mathfrak{d})$ for 
large $i$, in the range where $H^{i, j+1}(g)=H^{i+1, j}(g)=0$ (we can assume $H^{i, *}(g)=0$ for $\left.i \geq l\right)$. Hence $H^{i, j}(\mathfrak{d})=0$ for $i \gg 0$, cf. [23, Theorem 16]. Thus $\operatorname{dim} \mathfrak{d}_{k}$ grows polynomially for $k \gg 0$ and this implies that $h_{k}$ grows polynomially in the same range, whence the claim.

Let us note that we have not used Lie-Tresse theorem for the generalized equation $\mathcal{E}$ in this proof, but it generalizes to this case as well.

Now we derive a version of Arnold's strong conjecture.

Theorem 3. Let an algebraic pseudogroup $G$ act transitively on a manifold $M$ and its prolonged action preserve an algebraic differential equation $\mathcal{E} \subset J_{n}^{\infty}(M)$. Then there exist a subset $\Sigma \subset \mathcal{E}$ of $\operatorname{codim} \Sigma=\infty$ and an algebraic stratification $\mathcal{E} \backslash \Sigma=\cup_{\alpha} \mathcal{E}_{\alpha}$ such that for every $\alpha$ the Poincaré function $P_{\alpha}(z)=P\left(a_{\infty} ; z\right)$ is rational with the only pole at $z=1$ of degree $d \leq n$. This $P_{\alpha}$ depends only on $\alpha$ and not on $a_{\infty} \in \mathcal{E}_{\alpha}$.

Note that a differential equation (taken together with all prolongations) $\mathcal{E}$ is itself of infinite codimension in $J_{n}^{\infty}(M)$ unless $\mathcal{E}=J_{n}^{\infty}(M)$, but codimension of $\Sigma$ is measured in $\mathcal{E}$.

Proof. Let us begin with $\mathcal{E}$. By Theorem 2 the Poincaré function $P(z)=P\left(a_{\infty} ; z\right)$ is of the required type as long as $a_{\infty}$ belongs to a Zariski open set $\mathcal{E}^{\prime \prime}$. The complement $\tilde{\mathcal{E}}=\mathcal{E} \backslash \mathcal{E}^{\prime \prime}$ is a Zariski closed subset of $\mathcal{E}$. If it is of infinite codimension, we are done. Otherwise it is a stratified algebraic generalized equation invariant under the action of $G$, and the assumptions of Theorem 2 are satisfied (in particular, the action is transitive on the base). It can happen that in addition to equalities, specifying $\mathcal{E} \subset \overline{\mathcal{E}}$ in the preceding proof, we introduce inequalities, but the conclusion will not suffer from this. Thus we apply Theorem 2 again and obtain rationality of the Poincare function on a Zariski open subset $\tilde{\mathcal{E}}^{\prime \prime}$ of $\tilde{\mathcal{E}}$. Continuing in the same way for at most countably many steps, we conclude the claim.

Let us give an example of a situation, where assumptions of the previous theorem fail and the conclusion is different. Consider vector fields on a manifold $N$ as sections of its tangent bundle. Here $M=T N$, $n=\operatorname{dim} N$, and we consider only jets of sections of $\pi: E=M \rightarrow$ $N$, restricting to $J^{\infty}(N, T N) \subset J_{n}^{\infty}(M)$. The group $G=\operatorname{Diff}_{\text {loc }}(N)$ naturally (and algebraically) acts on this jet-space. However $G$ does not act transitively on $M$ : there is an open orbit $U=T N \backslash N$ and the zero section $0_{N} \equiv N$. In the preimage $\pi_{\infty, 0}^{-1}(U)$ the Poincaré function is rational. In fact, it equals $P(z)=0$.

However for the points $a_{\infty}$ with $a=\pi_{\infty, 0}\left(a_{\infty}\right) \in 0_{N}$ the normal form theory applies, and $P\left(a_{\infty} ; z\right)$ depends essentially on the jet $a_{\infty}$. In this 
case, Arnold's conjecture is plausible, but the Poincaré function varies with $a_{\infty}$ : in non-resonant case (depends on $a_{1}$ only) the vector field is formally linearizable and so $P(z)=n z$, while the resonant formal normal form can lead to poles at other points on the unit circle $|z|=1$. We will discuss this phenomenon closer in the Conclusion.

\subsection{An example of computation. Consider the action}

$$
g:(x, y, u) \mapsto\left(X(x, y), y+c_{1}, u+c_{2}\right)
$$

of the pseudogroup $G=\{g\}$ on $M=\mathbb{R}^{3}(x, y, u)=\mathbb{R}^{2}(x, y) \times \mathbb{R}^{1}(u)$ and prolong it to $J^{\infty}\left(\mathbb{R}^{2}, \mathbb{R}\right)=\mathbb{R}^{\infty}\left(x, y,\left\{u_{i, j}\right\}_{i, j \geq 0}\right)$, where $u_{i, j}$ is the jet-coordinate corresponding to $D_{x}^{i} D_{y}^{j} u(x, y)$. The Lie algebra sheaf of $G$ is $\mathcal{G}=\left\langle f(x, y) \partial_{x}, \partial_{y}, \partial_{u}\right\rangle$. Note that the action is algebraic and transitive on the base, so all assumptions are satisfied.

The isotropy subalgebra in $\mathcal{G}$ of the point $a=0$ in $M$ is $\mathcal{G}_{a}=\{X=$ $\left.f(x, y) \partial_{x}: f(0,0)=0\right\}$. Note that prolongation of such $X$ to $J^{\infty}$ is

$$
X_{a}^{(\infty)}=-\sum_{i+j>0} D_{x}^{i} D_{y}^{j}\left(f(x, y) u_{10}\right) \partial_{u_{i, j}} .
$$

This action has a unique open orbit - the complement of the stratum $\Sigma_{1}=\left\{u_{10}=0\right\}$, i.e. $P(z)=0$ on $J^{\infty} \backslash \Sigma_{1}$. Indeed, the prolonged field to $k$-jets is

$$
X_{a}^{(k)}=-\sum_{0<i+j \leq k}\left(f_{i, j} u_{10}+\ldots\right) \partial_{u_{i, j}},
$$

where dots denote the lower jets of the group parameter $f$. Varying these jets makes the coefficients of $\partial_{u_{i, j}}$ arbitrary provided $u_{10} \neq 0$. Thus the orbit in $J^{k}$ is open, and all differential invariants are constants.

Consider the singular stratum $\Sigma_{1}=\left\{u_{10}=0\right\}$ (codimension 1, no prolongations). In this case

$$
X_{a}^{(k)}=-\sum_{1<i+j \leq k}\left(i f_{i-1, j} u_{20}+j f_{i, j-1} u_{11}+\ldots\right) \partial_{u_{i, j}}
$$

where dots denote the lower jets of $f$. Counting the group parameters we see that if $u_{20} \neq 0$ there is one pure order differential invariant in every order: $h_{k}=1$ for $k>0$. The first invariants are:

$$
I_{1}=u_{01}, I_{2}=u_{02}-\frac{u_{11}^{2}}{u_{20}}, I_{3}=u_{03}-\frac{u_{11}^{3}}{u_{20}^{3}} u_{30}+3 \frac{u_{11}^{2}}{u_{20}^{2}} u_{21}-3 \frac{u_{11}}{u_{20}} u_{12} .
$$

The next singular stratum is $\Sigma_{2}=\left\{u_{10}=0, u_{20}=0\right\}$. In this case a similar argument implies that provided $u_{11} \neq 0$ there is one pure order 
differential invariant $h_{k}=1$ in every order $0<k \neq 2$, and for $k=2$ we have $h_{2}=0$. The first invariants are:

$$
I_{1}=u_{01}, I_{3}=\frac{u_{30}}{u_{11}^{3}}, I_{4}=\frac{u_{40}}{u_{11}^{4}}-6 \frac{u_{30} u_{21}}{u_{11}^{5}}+3 \frac{u_{02} u_{30}^{2}}{u_{11}^{6}} .
$$

The next singular stratum is $\Sigma_{3}=\left\{u_{10}=0, u_{20}=0, u_{11}=0\right\}$. Here the same argument implies that provided $u_{30} \neq 0$ we have $h_{1}=h_{2}=1$ and $h_{k}=2$ for $k>2$, so that we obtain two new invariants in every order starting from order three. The first invariants are:

$I_{1}=u_{01}, I_{2}=u_{02}, I_{3 a}=u_{03}+2 \frac{u_{21}^{3}}{u_{30}^{2}}-3 \frac{u_{21} u_{12}}{u_{30}}, I_{3 b}=\frac{\left(u_{30} u_{12}-u_{21}^{2}\right)^{3}}{u_{30}^{4}}$.

In the same way we obtain all further singular strata $\Sigma_{4}=\left\{u_{10}=\right.$ $\left.0, u_{20}=0, u_{11}=0, u_{30}=0\right\}, \Sigma_{5}=\left\{u_{10}=0, u_{20}=0, u_{11}=0, u_{30}=\right.$ $\left.0, u_{21}=0\right\}, \Sigma_{6}=\left\{u_{10}=0, u_{20}=0, u_{11}=0, u_{30}=0, u_{21}=0, u_{12}=0\right\}$, etc. In the limit we get the stratum $\Sigma_{\infty}=\left\{u_{1+i, j}=0: i, j \geq 0\right\}$, which is the infinitely prolonged equation $\left\{u_{x}=0\right\}$. In this latter stratum the group reduces to three translations on the base, so all jetcoordinates $u_{01}, u_{02}, u_{03}, \ldots$ are differential invariants. We summarize our computations in the following table:

\begin{tabular}{|c|l|}
\hline$\Sigma_{0} \backslash \Sigma_{1}$ & $P(z)=0$ \\
$\Sigma_{1} \backslash \Sigma_{2}$ & $P(z)=z+z^{2}+z^{3}+\cdots=\frac{z}{1-z}$ \\
$\Sigma_{2} \backslash \Sigma_{3}$ & $P(z)=z+z^{3}+z^{4}+z^{5}+\cdots=\frac{z-z^{2}+z^{3}}{1-z}$ \\
$\Sigma_{3} \backslash \Sigma_{4}$ & $P(z)=z+z^{2}+2 z^{3}+2 z^{4}+2 z^{5}+\cdots=\frac{z+z^{3}}{1-z}$ \\
$\Sigma_{4} \backslash \Sigma_{5}$ & $P(z)=z+z^{2}+z^{3}+2 z^{4}+2 z^{5}+2 z^{6}+\cdots=\frac{z+z^{4}}{1-z}$ \\
$\Sigma_{5} \backslash \Sigma_{6}$ & $P(z)=z+z^{2}+z^{4}+z^{5}+z^{6} \cdots=\frac{z-z^{3}+z^{4}}{1-z}$ \\
$\Sigma_{6} \backslash \Sigma_{7}$ & $P(z)=z+z^{2}+z^{3}+3 z^{4}+3 z^{5}+3 z^{6}+\cdots=\frac{z+2 z^{4}}{1-z}$ \\
$\ldots$ & $\ldots \quad \cdots$ \\
$\Sigma_{\infty}$ & $P(z)=z+z^{2}+z^{3}+z^{4}+z^{5}+\cdots=\frac{z}{1-z}$ \\
\hline
\end{tabular}

The orbit foliation can have complicated singularities. Let us demonstrate this on example of the stratum $\Sigma_{1}$. For a point $a$ in it consider $\mathbb{R}^{3}=\pi_{2,1}^{-1}\left(a_{1}\right)$, with coordinates $r=u_{20}, s=u_{11}, t=u_{02}$. From formula (6) (in this case there will be no dots and summation is by $i+j=2)$, the Lie algebra sheaf $\mathcal{G}$ on it is given by two vector fields $X=2 r \partial_{r}+s \partial_{s}, Y=r \partial_{s}+2 s \partial_{t}$ (coefficients of $f_{10}$ and $f_{01}$ respectively).

The distribution $\langle X, Y\rangle$ is involutive and its foliation is shown below. The stratification is as follows: $r \neq 0$ (invariant $\left.I=t-\frac{s^{2}}{t}\right), r=0, s \neq 0$ (no invariants), $r=s=0$ (invariant $I=t$ ). 


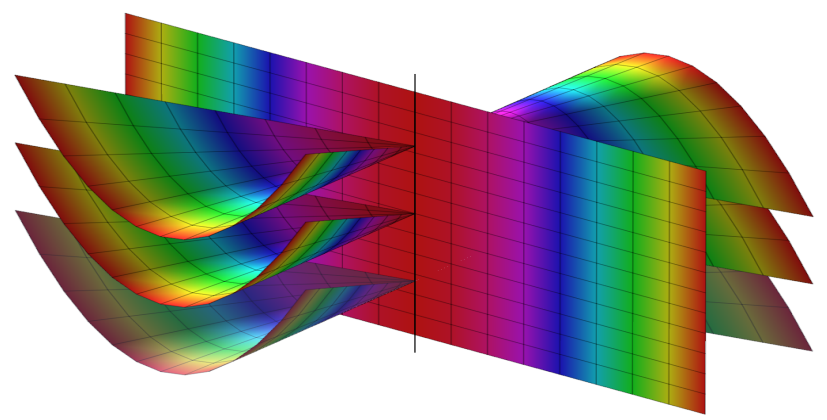

\section{On Computation of the Poincaré function}

In this and next sections we consider a natural bundle $\pi: E \rightarrow$ $M$, and $E$ (or an open subset of it) will play the role of $M$ from the previous section. From now on $M$ will be the base of the bundle $\pi$, and $G \subseteq \operatorname{Diff}_{\text {loc }}(M)$ a pseudogroup on $M$. In what follows an equality will be our primary interest (the other cases will be explicitly specified), so we specify the method to this case. Note that, by naturality of $\pi$, the action of $G$ lifts from $M$ to $E$, and hence $G \varsubsetneqq \operatorname{Diff}_{\text {loc }}(E)$.

Denote by $\mathbb{D}_{a}^{k}=\left\{[\varphi]_{a}^{k}: \varphi \in G=\operatorname{Diffloc}_{\mathrm{loc}}(M), \varphi(a)=a\right\}$ the socalled differential group of order $k$ on $M$ at $a$. If the above lift involves $r$ differentiations, i.e. the $G$-action on $E$ has order $r$, then $\mathbb{D}_{a}^{k+r}$ acts on $J_{a}^{k} \pi$. In almost all our examples the action will be transitive on $E=J^{0} \pi$ (the opposite will be explicitly noted). Moreover the lift from $M$ to $E$ and further prolongations will keep the action algebraic, so the assumptions of the previous section hold.

In some cases, we deal with pure jets, but in some others a differential equation is given, restricting the action to $\mathcal{E}_{a}^{k} \subseteq J_{a}^{k} \pi$ (un-restricted case corresponds to the equality). Thus, abbreviating $T_{a}=T_{a} M$, the fiber $\Delta_{k+r} \simeq S^{k+r} T_{a}^{*} \otimes T_{a}$ of the projection $\mathbb{D}_{a}^{k+r} \rightarrow \mathbb{D}_{a}^{k+r-1}$ over the unit (identity) acts on the symbol $g_{k}\left(a_{k}\right)=T_{a_{k}} \mathcal{E}^{k} \rightarrow T_{a_{k-1}} \mathcal{E}^{k-1}$; here $a_{k}$ is a sequence of jets in $\mathcal{E}_{a}^{k}$ with projective limit $a_{\infty}$, i.e. $\pi_{k, k-1} a_{k}=a_{k-1}$.

Note that if $X$ is a vector field vanishing to order $k+r$ at $a$ and $s$ is a (local) section of $\pi$, then $L_{X}(s)$ vanishes to order $k$ at $a$. Denoting $\lambda=[X]_{a}^{k+r} \in \Delta_{k+r}$ the corresponding jet and $\rho: S^{r} T_{a}^{*} \otimes T_{a} \rightarrow T_{a_{0}} E_{a}$ the symbol of the action, we have for a section with $s(a)=a_{0}:\left[L_{X} s\right]_{a}^{k}=$ $\zeta_{a_{0}}^{k}(\lambda)$, where $\zeta_{a_{0}}^{k}$ is the composition of the canonical splitting map $\delta_{k}$ and the prolongation of the symbol map,

$$
\zeta_{a_{0}}^{k}: S^{k+r} T_{a}^{*} \otimes T_{a} \stackrel{\delta_{k}}{\longrightarrow} S^{k} T_{a}^{*} \otimes S^{r} T_{a}^{*} \otimes T_{a} \stackrel{1 \otimes \rho}{\longrightarrow} S^{k} T_{a}^{*} \otimes T_{a_{0}} E_{a}
$$


This implies the following. Denote $\mathfrak{g}_{r}=\operatorname{Ker}\left(\rho: S^{r} T_{a}^{*} \otimes T_{a} \rightarrow T_{a_{0}} E_{a}\right)$ and let $\mathfrak{g}_{k+r}=\mathfrak{g}_{r}^{(k)}=S^{k} T_{a}^{*} \otimes \mathfrak{g}_{r} \cap S^{k+r} T_{a}^{*} \otimes T_{a}$ be its prolongation (to complete this symbolic system, we let $\mathfrak{g}_{i}=S^{i} T_{a}^{*} \otimes T_{a}$ for $\left.0 \leq i<r\right)$.

Proposition 4. The tangent space to the orbit $\Delta_{k+r}\left(a_{k}\right) \subset T_{a_{k}} \mathcal{E}^{k}$ is equal to the image $\operatorname{Im}\left(\zeta_{a_{0}}^{k}\right)$. Moreover, $\operatorname{Ker}\left(\zeta_{a_{0}}^{k}\right)=\mathfrak{g}_{k+r}$ and the normal space to the orbit is $\operatorname{CoKer}\left(\zeta_{a_{0}}^{k}\right)$.

For $r=1$ the map $\delta=\delta_{1}$ is the usual Spencer differential and the above prolongation is the standard Sternberg prolongation of first order structures. Note that in presence of equation $\mathcal{E}$ the size of kernel and cokernel of the map $\zeta_{a_{0}}^{k}$ may vary, we will comment in such cases.

In the case of Riemannian structures, when $r=1$ and $\mathfrak{g}=\mathfrak{s o}(n)$, the above proposition was proved in [30]. In this case $\mathfrak{g}_{i}=0$ for $i \geq 2$. In the case of symmetric connections (considered in details in the next section), $r=2$ and the symbol $\rho: S^{2} T_{a}^{*} \otimes T_{a} \rightarrow S^{2} T_{a}^{*} \otimes T_{a}$ is an isomorphism, whence $\mathfrak{g}_{i}=0$ for $i \geq 2$.

Denote by $\mathrm{St}_{a_{k}}^{k+r} \subset \mathbb{D}_{a}^{k+r}$ the connected component of unity in the stabilizer of $a_{k} \in \mathcal{E}_{a}^{k}$. We have: $\operatorname{Ker}\left(d \pi_{k+r, k+r-1} \mid \mathrm{St}_{a_{k}}^{k+r}\right)=\mathrm{St}_{a_{k}}^{k+r} \cap \Delta^{k+r}=$ $\operatorname{Ker}\left(\zeta_{a_{0}}^{k}\right)$. Then Proposition 4 implies:

Corollary. Assume that equation $\mathcal{E}$ is regular. If $\mathfrak{g}$ is of finite type and $\mathfrak{g}_{i}=0$ for $i \geq l$, then $\operatorname{Ker}\left(\zeta_{a_{0}}^{k}\right)=0$ for $k \geq l-r$. Consequently, the projection $\pi_{k+r, k+r-1}: \mathrm{St}_{a_{k}}^{k+r} \rightarrow \mathrm{St}_{a_{k-1}}^{k+r-1}$ is injective for such $k$.

The action is locally free from the jet-level $l$ if $\mathrm{St}_{a_{k}}^{k+r}=0$ for $k \geq l$ and Zariski-generic $a_{k}$. Note that in all cases of finite type we consider in the next section, the stabilizer will be resolved in a finite number of prolongations. This has the following explanation. Since the Lie equation is of finite type at generic jet of the geometric structure, stabilization of $\mathrm{St}_{a_{k}}^{k+r}$ at non-zero space would imply non-trivial local symmetry of the considered structure, while in all our examples generic geometric structures will have only trivial local symmetries.

\section{A PANORAMA OF EXAMPLES}

Below we compute the Poincaré function of most popular geometric structures, whose moduli arise in applications. Some of these formulae have been known before and we provide a reference, for some others only the orbit dimensions have been known and we derive a compact formula for the Poincaré series (usage of Maple is acknowledged at that stage). We also correct errors in several previous works on the subject, and provide short computations based on prolongation technique of the previous section. Finally, we add new examples: metric connections, 
Weyl conformal and almost complex structures. The latter case is especially interesting as the first non-trivial structure of infinite type with novel effects in local moduli count.

We denote $n=\operatorname{dim} M$ in all computations in this section, unless otherwise specified (dimension $d$ if $M$ will be indicated as $M^{d}$ ).

3.1. Second order ODE modulo point transformations. This is one of the most known classical examples, where differential invariants have been computed and counted. Our only contribution here are the formulae for the Poincaré function.

3.1.1. General second order ODE. Consider differential equations $y^{\prime \prime}=f\left(x, y, y^{\prime}\right)$ given by a function $f$ of three variables. The action of the pseudogroup $G=\operatorname{Diff}_{\text {loc }}\left(\mathbb{R}^{2}\right)$ on the space of independent and dependent variables $(x, y)$ prolongs to the space $\mathbb{R}^{4}\left(x, y, y^{\prime}, y^{\prime \prime}\right)$. In this case $M=\mathbb{R}^{2}$ and $E=\mathbb{R}^{4}$. Denoting $y^{\prime}=p, y^{\prime \prime}=u$, we get a transitive algebraic action on the space $J^{0}=\mathbb{R}^{3}(x, y, p) \times \mathbb{R}^{1}(u)$, which prolongs to the action on the space $J^{\infty}\left(\mathbb{R}^{3}\right)$ of jets of functions $u=f(x, y, p)$.

The problem of differential invariants of this action was initiated by S. Lie [27], and all relative invariants were found by A. Tresse [40]. The absolute differential invariants were derived and counted in [16]: $h_{k}=0$ for $k \leq 4, h_{5}=3$ and $h_{k}=\left(\begin{array}{l}k \\ 2\end{array}\right)-4$ for $k>5$. Therefore we obtain

$$
P(z)=3 z^{5}+\sum_{k=5}^{\infty}\left(\frac{k(k-1)}{2}-4\right) z^{k}=\frac{z^{5}\left(3+2 z-7 z^{2}+3 z^{3}\right)}{(1-z)^{3}} .
$$

There are no differential invariants of order up to four: $G$ acts transitively on $J^{3}$, and has a Zariski open orbit in $J^{4}$ - its complement is a reducible algebraic variety $I \cdot H=0$, where $I, H$ are basic relative invariants. The numbers $s_{k}=\sum_{i<k} h_{i}$ correspond to codimension of the orbit in the domain $I \neq 0, H \neq 0$ of $k$-jets.

3.1.2. Second order ODE cubic in $y^{\prime}$. The singular stratum given by $H=0$ is dual by E. Cartan [6] to $I=0$, so it is enough to consider only the latter. This relative invariant has a simple formula $I=\frac{\partial^{4} f}{\partial p^{4}}$, so $I=0$ is equivalent to cubic dependence of the right-hand side of the ODE on $p=y^{\prime}: y^{\prime \prime}=\alpha_{0}(x, y)+\alpha_{1}(x, y) y^{\prime}+\alpha_{2}(x, y)\left(y^{\prime}\right)^{2}+\alpha_{3}(x, y)\left(y^{\prime}\right)^{3}$. Such ODEs are equivalent to projective connections in 2D [6].

The group $G$ acts on $J^{0}=\mathbb{R}^{2}(x, y) \times \mathbb{R}^{4}\left(\alpha_{0}, \alpha_{1}, \alpha_{2}, \alpha_{3}\right)$ and the action prolongs to $J^{\infty}\left(\mathbb{R}^{2}, \mathbb{R}^{4}\right)$. This action is transitive in 2 -jets, and transitive outside the stratum $F_{3}=0$ in 3-jets, where $F_{3}$ is the Liouville relative invariant [29], see also [16]. Differential invariants of this action 
were counted in [39, 44]: $h_{k}=0$ for $k<4, h_{k}=2(k-1)$ for $k \geq 4$. Therefore we obtain

$$
P(z)=\sum_{k=4}^{\infty} 2(k-1) z^{k}=\frac{2 z^{4}(3-2 z)}{(1-z)^{2}} .
$$

3.1.3. Second order ODEs of special Lie form. The following class of equations was introduced by S. Lie: $y^{\prime \prime}=f(x, y)$. It includes all Painlevé transcendents (after a point transformation [5]) and so is of special importance. The point transformation pseudogroup leaving the class invariant is $(x, y) \mapsto\left(X(x), c X^{\prime}(x)^{1 / 2} y+Y(x)\right)$, it naturally extends to the space $J^{0}=\mathbb{R}^{2}(x, y) \times \mathbb{R}(f)$. Differential invariants of this action were computed by P. Bibikov [4]. In particular, $h_{k}=0$ for $k \leq 3$, $h_{4}=2$, and $h_{k}=k-1$ for $k \geq 5$. This implies the formula

$$
P(z)=2 z^{4}+\sum_{k=5}^{\infty}(k-1) z^{k}=\frac{z^{4}\left(2-z^{2}\right)}{(1-z)^{2}} .
$$

3.2. Metric and related structures. Consider $E=S^{2} T^{*} M$. The group $G$ acts in the fiber $S^{2} T_{x}^{*} M$ through the general linear group $G L\left(T_{x} M\right)$. The action is not transitive, and degenerate quadrics form a singular stratum $\Sigma_{x}$. The complement to $\Sigma=\cup_{x} \Sigma_{x}$ in $E$ is one orbit of $G=\operatorname{Diff}_{\text {loc }}(M)$ over $\mathbb{C}$, while over $\mathbb{R}$ it splits into a finite union of orbits numerated by the index. Resetting $E$ to be one of those domains we get an algebraic fiber bundle on which $G$ acts transitively. Sections of it correspond to (pseudo-)Riemannian metrics $g$. Note that the number of differential invariants is independent of the index of $g$ and so the Poinaré function is the same for pseudo-Riemannian metrics as for Riemannian ones.

Below we study a bundle of metrics or a differential equation in it; we also impose additional a complex or tri-complex structure, constrained by the known relations. The bundle $E$ is properly modified.

3.2.1. Riemannian metrics on $M^{n}$. Local scalar differential invariants of metrics for $n=2$ were studied by K. Zorawski [45], and their count is: $h_{k}=0$ for $k<2, h_{2}=h_{3}=1, h_{k}=k-1$ for $k>3$.

When $n>2$, the count of invariants was done by C. N. Haskins [12]: $h_{k}=0$ for $k<2, h_{2}=\frac{1}{2}\left(\begin{array}{l}n \\ 3\end{array}\right)(n+3)$ and $h_{k}=\left(\begin{array}{c}n+1 \\ 2\end{array}\right)\left(\begin{array}{c}n+k-1 \\ k\end{array}\right)-n\left(\begin{array}{c}n+k \\ k+1\end{array}\right)$ for $k>2$. This implies the formula [18]:

$$
P(z)= \begin{cases}\frac{z^{2}\left(1-z+2 z^{2}-z^{3}\right)}{(1-z)^{2}}, & \text { for } n=2 \\
\frac{n}{z}+\left(\begin{array}{c}
n \\
2
\end{array}\right) \cdot\left(1-z^{2}\right)-\frac{1}{(1-z)^{n}} \cdot\left(\frac{n}{z}-\left(\begin{array}{c}
n+1 \\
2
\end{array}\right)\right), & \text { for } n>2 .\end{cases}
$$


Note that singularity at $z=0$ is inessential and is used here (and below) for compactification of the answer.

3.2.2. Einstein metrics. This is an important special stratum. Note that the Einstein condition $\operatorname{Ric}_{g}=\Lambda g$ for some $\Lambda \in C^{\infty}(M)$ is an equation $\mathcal{E}$ on the sections of the bundle $E$ from the general case. Recall that $\Lambda$ is constant and non-trivial cases arise for $n \geq 4$ (indeed, $P(z)=z^{2}$ for $\left.n=2,3\right)$. The description and count of differential invariants in the 4D case was done by V. Lychagin and V. Yumaguzhin [30]. Their method extends further, as follows.

Proposition 5. We have: $h_{k}=0$ for $k<2, h_{2}=\frac{1}{12}\left(n^{2}-1\right)\left(n^{2}-12\right)$ and $h_{k}=\frac{(k-1) n(n+k-1)(n+2 k-2)}{2(k+1)(n-2)}\left(\begin{array}{c}n+k-4 \\ k\end{array}\right)$ for $k>2$.

Proof. In this case $r=1, a_{0}=g$ is a (pseudo-) Riemannian metric and similar to [30] (beware of different indexing convention for stabilizers) we compute $\mathrm{St}_{a_{1}}^{2} \simeq \mathrm{St}_{a_{0}}^{1}=S O(g)$. Since $a_{2}$ encodes the curvature tensor that, for Einstein metrics, consists of the scalar curvature and the Weyl tensor, for generic $a_{2}$ and $n \geq 4, k>1$ we get $\mathrm{St}_{a_{k}}^{k+1}=0$. Thus the action is locally free from the jet-level 2 .

Now we can easily compute the orbit dimensions: the orbit of $\mathbb{D}_{a}^{k+1}$ in $\mathcal{E}_{a}^{k}$ has dimension of $\mathcal{E}_{a}^{k}=J_{a}^{k}$ for $k \leq 1$ and it has dimension of $\mathbb{D}_{a}^{k+1}$ for $k \geq 2$. Below we use the formulae $\operatorname{dim} S^{k} T_{a}^{*}=\left(\begin{array}{c}n+k-1 \\ k\end{array}\right)$, $\operatorname{dim} \oplus_{i \leq k} S^{i} T_{a}^{*}=\left(\begin{array}{c}n+k \\ k\end{array}\right)$, in particular $\operatorname{dim} \mathbb{D}_{a}^{k}=n\left(\begin{array}{c}n+k \\ k\end{array}\right)$.

The Einstein equation $\mathcal{E}$ is expressed by $\left(\begin{array}{c}n+1 \\ 2\end{array}\right)-1$ second-order conditions (traceless Ricci tensor vanishes). Consider at first the Ricci-flat equation, whose symbol $\sigma_{\text {Ric }}$ is resolved via the following acyclic complex (see [3, 17, 30])

$$
0 \rightarrow g_{k} \longrightarrow S^{k} T_{a}^{*} \otimes S^{2} T_{a}^{*} \stackrel{\sigma_{\mathrm{Ric}}}{\longrightarrow} S^{k-2} T_{a}^{*} \otimes S^{2} T_{a}^{*} \stackrel{\sigma_{\mathrm{Bn}}}{\longrightarrow} S^{k-3} T_{a}^{*} \otimes T_{a}^{*} \rightarrow 0
$$

in which $\sigma_{\mathrm{Bnc}}$ is the symbol of the Bianchi operator and $g_{k}$ is the symbol of $\mathcal{E}$, i.e. $\operatorname{Ker}\left(T \mathcal{E}_{a}^{k} \rightarrow T \mathcal{E}_{a}^{k-1}\right)$. Thus for the Ricci flat equation we get

$\operatorname{dim} g_{k}=\left(\begin{array}{c}n+k-1 \\ k\end{array}\right)\left(\begin{array}{c}n+1 \\ 2\end{array}\right)-\left(\begin{array}{c}n+k-3 \\ k-2\end{array}\right)\left(\begin{array}{c}n+1 \\ 2\end{array}\right)+\left(\begin{array}{c}n+k-4 \\ k-3\end{array}\right) n$

(we let $\left(\begin{array}{c}m \\ k\end{array}\right)=0$ for $k<0$ ). The only difference for Einstein equation is that we change $\operatorname{dim} g_{2}=\left(\begin{array}{c}n+1 \\ 2\end{array}\right)^{2}-\left(\begin{array}{c}n+1 \\ 2\end{array}\right)$ to $\operatorname{dim} \bar{g}_{2}=\operatorname{dim} g_{2}+1$.

This implies $h_{0}=h_{1}=0$ and $h_{2}=\operatorname{dim} \bar{g}_{2}-\operatorname{dim} \Delta_{3}-\operatorname{dim} S O(g)=$ $\frac{1}{12} n^{2}\left(n^{2}-13\right)+1$ (the stabilizer is resolved at this step). For $k>2$ we obtain $h_{k}=\operatorname{dim} g_{k}-\operatorname{dim} \Delta_{k+1}$ and the result follows. 
This proposition implies the formula

$$
P(z)=\frac{n(z+1)\left((n+1) z-2\left(z^{2}+1\right)\right)}{2 z(1-z)^{n-1}}-\left(\begin{array}{c}
n \\
2
\end{array}\right)\left(z^{2}-1\right)+\frac{n}{z}+z^{2} .
$$

For physically relevant case of Lorntzian metrics in $4 \mathrm{D}$ this formula simplifies to the following, where the first term in the last expression is the Poincaré function for Ricci-flat 4D metric derived in [30].

$$
P(z)=\frac{z^{2}\left(5+9 z-15 z^{2}+5 z^{3}\right)}{(1-z)^{3}}=\frac{2 z^{2}\left(2+6 z-9 z^{2}+3 z^{3}\right)}{(1-z)^{3}}+z^{2} .
$$

3.2.3. Self-dual metrics in $4 \mathrm{D}$. This is another important special stratum. The self-duality condition $* W_{g}=W_{g}$ is an equation $\mathcal{E}$ on the sections of the bundle $E$ from the general case.

The description and count of differential invariants in this case was done by the author and E. Schneider [20]: $h_{k}=0$ for $k<2, h_{2}=9$ and $h_{k}=\frac{1}{6}(k-1)\left(k^{2}+25 k+36\right)$ for $k>2$. This implies the formula for the Poincaré function [20]:

$$
P(z)=\frac{z^{2}\left(9+4 z-30 z^{2}+24 z^{3}-6 z^{4}\right)}{(1-z)^{4}} .
$$

3.2.4. Kähler metrics on $M^{2 n}$. Though considered as metrics, they are not a stratum in the space of metrics. Indeed, a Kähler structure is given by the first order equation $\mathcal{E}=\left\{\nabla^{g} J=0\right\}$ on the bundle of almost Hermitian pairs $(g, J)$ over $M$, the algebraic constraints are: $J^{2}=-\mathbf{1}, J^{*} g=g$. The signature of $g$ does not influence the computation below, which thus applies to pseudo-Kähler structures as well.

The count of differential invariants in this case was done by $\mathrm{A}$. Schmelev [35]: $h_{k}=0$ for $k<2, h_{2}=\frac{1}{4} n^{2}(n-1)(n+3)$ and $h_{k}=\left(\begin{array}{c}2 n+k+1 \\ k+2\end{array}\right)-2\left(\begin{array}{c}n+k+1 \\ k+2\end{array}\right)-2 n\left(\begin{array}{l}n+k \\ k+1\end{array}\right)$ for $k>2$. Note that though dimensions $s_{k}=\sum_{i=0}^{k} h_{i}$ are correct in [35], the sequence $h_{k}$ in the Poincaré series in Theorem 2.12 of loc.cit. has a flaw (wrong coefficient $h_{2}$ at $z^{2}$ ). The proper formula is (note also that the case $n=1$ is special, since the equation $\mathcal{E}$ is trivial and a Kähler metric is identical to a $2 \mathrm{D}$ Riemannian metric and an orientation):

$$
P(z)= \begin{cases}\frac{z^{2}\left(1-z+2 z^{2}-z^{3}\right)}{(1-z)^{2}}, & \text { for } n=1 \\ \frac{1}{z^{2}(1-z)^{2 n}}-\frac{2(z n+1)}{z^{2}(1-z)^{n}}+n^{2}\left(1-z^{2}\right)+\frac{2 n z+1}{z^{2}}, & \text { for } n>1 .\end{cases}
$$

3.2.5. Hyper-Kähler metrics on $M^{4 n}$. Similarly, consider the equation $\mathcal{E}$ in the bundle of almost hyper-Hermitian structures $(g, I, J, K)$ given by conditions that the operator fields $I, J, K$ satisfy the quaternionic relations and are orthogonal with respect to $g$. The equation 
$\mathcal{E}$ describes integrability of $I_{a} \in\{I, J, K\}$ and closedness of the corresponding 2-forms $\omega_{a}=g\left(I_{a} \cdot, \cdot\right)$; equivalently the condition $\nabla_{g} I_{a}=0$ is imposed for all $a$. The pseudo-group is $G=\operatorname{Diff}_{\mathrm{loc}}(M)$, as before.

The dimensions $s_{k}$ were computed by A. Schmelev [35]. This implies: $h_{k}=0$ for $k<2, h_{2}=\frac{1}{6} n(n+3)(2 n-1)(2 n+1)$ and $h_{k}=$ $2 \sum_{i=0}^{n}\left(\begin{array}{c}2 n+k-i \\ k\end{array}\right)(n-i)-\left(\begin{array}{c}2 n+k+1 \\ k+2\end{array}\right)-2\left(\begin{array}{c}n+k+1 \\ k+2\end{array}\right)$ for $k>2$. However the coefficient $h_{2}$ at $z^{2}$ in the Poincaré series in Theorem 3.15 of loc.cit. is wrong, so the answer there is not correct. The proper formula is

$$
P(z)=\frac{2 n}{z(1-z)^{2 n+1}}-\frac{3}{z^{2}(1-z)^{2 n}}-n(2 n+1)\left(z^{2}-1\right)+\frac{4 n z+3}{z^{2}} .
$$

Note that for $n=1$ hyper-Kähler metrics are Ricci-flat self-dual metrics in $4 \mathrm{D}$, so this case is on an intersection of subsections 3.2 .2 and 3.2 .3 .

3.3. Linear connections. These are sections of the affine bundle $E$ associated with the vector bundle $T^{*} M \otimes T^{*} M \otimes T M$ (or $S^{2} T^{*} M \otimes T M$ for symmetric connections). Note that in general, the torsion $T_{\nabla}$ of a connection $\nabla$ is a 0 -th order invariant, so the action of $G=\operatorname{Diff}_{\text {loc }}(M)$ on $E$ is not transitive. The bundle $E$ is however algebraic and the conclusion of Theorem 1 holds true. Indeed, scalar differential invariants of 0 -th order are rational invariants of the general linear group on the space of torsion tensors (note that scalar polynomial differential invariants are only constants [11]). For $n \geq 3$ these also generate invariant derivations, whence a Lie-Tresse generation property (first order invariants should be used for $n=2$ to get this).

3.3.1. General linear connections on $M^{n}$. Since the connections for $n=1$ are all locally equivalent, we assume $n>1$. The dimensions $h_{k}$ were computed by T. Thomas [38, see also [9]:

Proposition 6. For $n>2$ we have: $h_{0}=\frac{1}{2} n^{2}(n-3)$ and $h_{k}=$ $n^{3}\left(\begin{array}{c}n+k-1 \\ k\end{array}\right)-n\left(\begin{array}{c}n+k+1 \\ k+2\end{array}\right), k>0$. In the exceptional case $n=2$ we get: $h_{0}=0, h_{1}=6, h_{k}=6 k+2, k>1$.

The computation is easy, we present a short independent argument.

Proof. Let us split $\nabla_{X} Y=\nabla_{X}^{0} Y+\frac{1}{2} T_{\nabla}(X, Y)$, where $T_{\nabla} \in \Lambda^{2} T_{a}^{*} \otimes T_{a}$ is torsion of the connection $\nabla$ and $\nabla^{0}$ is a symmetric connection. The tensor $T_{\nabla}$ is a first-order structure, i.e. $r=1$, and the action of the group $G=\operatorname{Diff}_{\text {loc }}(M)$ is locally free starting from the jet-level 0 for $n>2$ and starting from the jet-level 1 for $n=2$. The symmetric connection $\nabla^{0}$ has order $r=2$; it is not a tensor, but a section of an affine bundle with corresponding vector space $S^{2} T_{a}^{*} \otimes T_{a}$. The action of $G$ is locally free starting from the jet-level 1 . Indeed, the symbolic 
system associated to the action is the following: $\mathfrak{g}_{0}=T_{a}, \mathfrak{g}_{1}=\operatorname{End}\left(T_{a}\right)$ and $\mathfrak{g}_{i}=0$ for $i \geq 2$. Thus $\pi_{k+2,3}: \mathrm{St}_{a_{k}}^{k+2} \stackrel{\sim}{\rightarrow} \mathrm{St}_{a_{1}}^{3}=0$ for generic $a_{1}$.

Therefore in the case $n>2$ we get: $h_{0}=\operatorname{dim} g_{0}-\operatorname{dim} \Delta_{1}-\operatorname{dim} \Delta_{2}=$ $n^{3}-n^{2}-n\left(\begin{array}{c}n+1 \\ 2\end{array}\right)$ and for $k>0$

$$
h_{k}=\operatorname{dim} g_{k}-\operatorname{dim} \Delta_{k+2}=n^{3}\left(\begin{array}{c}
n+k-1 \\
k
\end{array}\right)-n\left(\begin{array}{c}
n+k+1 \\
k+2
\end{array}\right) .
$$

In the case $n=2$ the torsion has a unique non-zero orbit, so $h_{0}=0$ and the 2-dimensional stabilizer is resolved in 1-jets, whence $h_{1}=6$, $h_{k}=8(k+1)-2(k+3)=6 k+2$ for $k>1$.

We conclude for general linear connections (the Poincaré function was proved rational in [9], but the explicit form was not derived):

$$
P(z)= \begin{cases}\frac{2 z\left(3+z-z^{2}\right)}{(1-z)^{2}}, & \text { for } n=2 \\ \frac{n\left(n^{2} z^{2}-1\right)}{z^{2}(1-z)^{n}}-n^{2}+\frac{n(n z+1)}{z^{2}}, & \text { for } n>2 .\end{cases}
$$

3.3.2. Symmetric connections on $M^{n}$. This case, which is a singular stratum in the space of general connections, was investigated by $\mathrm{S}$. Dubrovskiy [7]. In his computations dimensions of the stabilizers were correctly determined, but dimensions $h_{k}$ were computed wrongly due to an arithmetic error (two flaws in Theorem 2.4 of loc.cit.: factor $n$ before the second binomial coefficient should be omitted, and $n^{2}$ shall be subtracted from the coefficient $h_{1}$ ). Correcting these yields:

Proposition 7. We have: $h_{0}=0, h_{1}=\frac{1}{3} n^{2}\left(n^{2}-4\right)+\delta_{2, n}, h_{2}=$ $n\left(\begin{array}{c}n+1 \\ 2\end{array}\right)^{2}-n\left(\begin{array}{c}n+3 \\ 4\end{array}\right)-\delta_{2, n}$, and $h_{k}=n\left(\begin{array}{c}n+1 \\ 2\end{array}\right)\left(\begin{array}{c}n+k-1 \\ n-1\end{array}\right)-n\left(\begin{array}{c}n+k+1 \\ n-1\end{array}\right)$ for $k \geq 2$.

Let us give some details (our computation is independent of [7]).

Proof. As we noted above, symmetric connections are structures of order $r=2$ and the action of $G=\operatorname{Diff}_{\text {loc }}(M)$ is locally free starting from the jet-level 1 for $n>2$. Thus

$$
h_{0}=\operatorname{dim} g_{0}-\operatorname{dim} \Delta_{2}=n\left(\begin{array}{c}
n+1 \\
2
\end{array}\right)-n\left(\begin{array}{c}
n+1 \\
2
\end{array}\right)=0 .
$$

Next $h_{1}=\operatorname{dim} g_{1}-\operatorname{dim} \Delta_{1}-\operatorname{dim} \Delta_{3}=\frac{1}{3} n^{2}\left(n^{2}-4\right)$ is the number of rational invariants of the (free) action of the general linear group $G L(n)$ on the space of curvature tensors $\mathcal{K}=\operatorname{Ker}\left(\Lambda^{2} T_{a}^{*} \otimes \mathfrak{g l}(n) \rightarrow \Lambda^{3} T_{a}^{*} \otimes T_{a}\right)$. For $k>1$ we get:

$$
h_{k}=\operatorname{dim} g_{k}-\operatorname{dim} \Delta_{k+2}=\frac{1}{2} n^{2}(n+1)\left(\begin{array}{c}
n+k-1 \\
k
\end{array}\right)-n\left(\begin{array}{c}
n+k+1 \\
k+2
\end{array}\right) \text {. }
$$


In the case $n=2$ the action of $G L(2)$ on the space of curvature (or Ricci) tensors is not free, there is a one-dimensional stabilizer that is resolved on the next jet-level. Thus here $h_{1}$ increases by 1 and $h_{2}$ decreases by 1 , implying the claim.

This implies the formula:

$$
P(z)= \begin{cases}\frac{z\left(1+5 z-z^{2}-z^{3}\right)}{(1-z)^{2}}, & \text { for } n=2, \\ \frac{\left(n(n+1) z^{2}-2\right) n}{2 z^{2}(1-z)^{n}}-n^{2} z+\frac{n(1+n z)}{z^{2}}, & \text { for } n>2 .\end{cases}
$$

3.3.3. Metric connections on $M^{n}$. A metric connection consists of a pair $(g, \nabla)$, where $g$ is a (pseudo-)Riemannian metric, $\nabla$ a linear connection on $M$, and $\nabla g=0$. The structure is the pair composed of a metric $g$, which has order $r=1$, and a connection $\nabla$, which has order $r=2$ with respect to the action of $G=\operatorname{Diff}_{\text {loc }}(M)$.

It is well known that given $g$ such $\nabla$ are bijective with their torsions $T=T_{\nabla} \in \Gamma\left(\Lambda^{2} T^{*} M \otimes T M\right)$. Indeed, $\alpha_{X}=\nabla_{X}-\nabla_{X}^{g}$ is a $g$-skew symmetric map for every $X \in T M$. The skew-symmetrization $\operatorname{map} T_{a}^{*} \otimes \Lambda^{2} T_{a}^{*} \rightarrow \Lambda^{2} T_{a}^{*} \otimes T_{a}^{*}$, given by $\alpha \mapsto \tau=\alpha_{\curlywedge}, \tau(X, Y, Z)=$ $\frac{1}{2}(\alpha(X, Y, Z)-\alpha(Y, X, Z))$, is an isomorphism; its inverse is given by the formula $\alpha(X, Y, Z)=\tau(X, Y, Z)-\tau(X, Z, Y)-\tau(Y, Z, X)$. Denote the inverse map $\tau \mapsto \alpha=\tau_{\curlyvee}$ : $\left(\tau_{\curlyvee}\right)_{\curlywedge}=\tau$. Using the operation $\sharp$ of raising the indices, we conclude the formula $\nabla-\nabla^{g}=\frac{1}{2} T_{\curlyvee}^{\sharp}$, i.e. $g\left(\nabla_{X} Y, Z\right)=$ $\left.g\left(\nabla_{X}^{g} Y, Z\right)+\frac{1}{2}(g(T(X, Y), Z)+g(T(Z, X), Y)-g(T(Y, Z), X))\right)$.

Thus we consider the pairs $(g, T)$, which are simpler objects but their jets are staggered: $k$-jet of $(g, \nabla)$ corresponds to $(k+1)$-jet of $g$ and $k$ jet of $T$ for $k>0$. Note that 1-st derivatives of $g$ are bijective with the Christoffel coefficients of $\nabla$. Indeed, lowering the indices $\Gamma_{i j k}=\Gamma_{i j}^{l} g_{l k}$, $T_{i j k}=T_{i j}^{l} g_{l k}$, one gets the relations $\partial_{k} g_{i j}=\Gamma_{i k j}+\Gamma_{j k i}$ and

$$
2 \Gamma_{i j k}=\partial_{i} g_{j k}+\partial_{j} g_{i k}-\partial_{k} g_{i j}+T_{i j k}+T_{j k i}-T_{k i j} \text {. }
$$

Thus 0-jet of our pair is given by the values of $g$ and $T$, while for $k>0$ the $k$-jet of the pair is bijective with $(k+1)$-jet of $g$ and $k$-jet of $T$.

Note that the bundle of metric connections is another instance where the pseudogroup $G$ acts non-transitively. However the same remarks as in subsection 3.3.1 apply here, and we can proceed as before. The count of invariants for the pairs $(g, T)$ is given as follows.

Proposition 8. We have: $h_{0}=\frac{n}{2}(n-1)^{2}$ and $h_{k}=\operatorname{dim} g_{k}-\operatorname{dim} \Delta_{k+1}=$ $\frac{n(n+1)}{2}\left(\begin{array}{l}n+k \\ k+1\end{array}\right)+\frac{n^{2}(n-1)}{2}\left(\begin{array}{c}n+k-1 \\ k\end{array}\right)-n\left(\begin{array}{c}n+k+1 \\ k+2\end{array}\right)$ for $k>0$.

Proof. The structure $(g, T)$ is a section of a tensor bundle of rank $\frac{1}{2} n\left(n^{2}+1\right)$. The general linear group acts on an open set of it freely 
(equivalently: the orthogonal group acts freely on the general stratum of the space of torsions) for $n>1$. Consequently, by the Corollary, the stabilizer $\mathrm{St}_{a_{k}}^{k+1}$ vanishes for all $k \geq 0$, and so the action is (locally) free from the jet-level 0 . The symbol $g_{k}$ of the structure, as a staggered pair $(g, T)$, is $S^{k+1} T_{a}^{*} \otimes S^{2} T_{a}^{*} \oplus S^{k} T_{a}^{*} \otimes \Lambda^{2} T_{a}^{*} \otimes T_{a}$ for $k \geq 1$.

Therefore $h_{k}=\operatorname{dim} g_{k}-\operatorname{dim} \Delta_{k+1}=\frac{n(n+1)}{2}\left(\begin{array}{c}n+k \\ k+1\end{array}\right)+\frac{n^{2}(n-1)}{2}\left(\begin{array}{c}n+k-1 \\ k\end{array}\right)-$ $n\left(\begin{array}{c}n+k+1 \\ k+2\end{array}\right)$, and the claim follows.

This implies (again we exclude the case $n=1$ as trivial: $P(z)=0$ ) the formula for $n \geq 2$ :

$$
P(z)=\frac{n-\left(\begin{array}{c}
n \\
2
\end{array}\right)\left(z^{2}-z\right)}{z^{2}}-\frac{2 n-n(n+1) z-n^{2}(n-1) z^{2}}{2 z^{2}(1-z)^{n}} .
$$

3.3.4. Metric connections with a skew-symmetric torsion. These form a partial case of general metric connections: these consist of $(g, T)$ with $T_{i j k}=T_{i j}^{l} g_{l k}$ being skew-symmetric in all indices. Since such $T$ vanishes in dimension $n=2$ (this case belongs to metrizable connections discussed below), so assume $n \geq 3$.

On the level of 0 -jets, the stabilizer of generic point $a_{0} \in \mathcal{E}_{a}^{0}$ corresponds to stabilizer of a generic 3-form in the orthogonal group, so the sequence $\mathrm{st}_{n}=\operatorname{dim} \mathrm{St}_{a_{0}}^{2}$, depending on $n=\operatorname{dim} M$, is the following: $\mathrm{st}_{3}=3, \mathrm{st}_{4}=3, \mathrm{st}_{5}=2$, and $\mathrm{st}_{n}=0$ for $n \geq 6$.

Remark. The stabilizers of a generic 3-form in the group $O(g)$ for a fixed metric $g$ on $T_{a}$, are: $S O(g)$ for $n=3, S O(3) \times \mathbb{Z}_{2}$ or $S O(1,2) \times \mathbb{Z}_{2}$ depending on the signature of $g$ for $n=4, S(O(2) \times O(2))$ for $n=5$, and trivial for $n \geq 6$. Note that extending $O(g)$ to $G L(n)$ the stabilizers become nontrivial for $n$ up to 8; for example, when $n=7$ the stabilizer of a generic 3-form $\omega$ is a real form of the exceptional Lie group $G_{2}$; it preserves some metric, depending on $\omega$, but not the given metric $g$.

Thus the action is free from the level of 0 -jets for $n \geq 6$ and from the level of 1 -jets for $3 \leq n \leq 5$. This implies

$$
P(z)=\frac{n-\left(\begin{array}{c}
n \\
2
\end{array}\right)\left(z^{2}-z\right)}{z^{2}}-\frac{n-\left(\begin{array}{c}
n+1 \\
2
\end{array}\right) z-\left(\begin{array}{c}
n \\
3
\end{array}\right) z^{2}}{z^{2}(1-z)^{n}}+\operatorname{st}_{n}(1-z) .
$$

The space of metrizable connections consists of such $\nabla$ that $T_{\nabla}=0$ and there exists a parallel metric $g$. Generic connections of this type have irreducible holonomy, and for them the metric $g$ is unique up to scale, i.e. $\nabla=\nabla^{g}$ is the Levi-Civita connection (for generic $\nabla$ the scale can be fixed by the requirement $\left\|R_{\nabla}\right\|_{g}^{2}= \pm 1$ ). Thus for metrizable connections the Hilbert function is equal to $h_{k+1}$ of subsection 3.2.1. 
and hence the Poincaré function of our problem is expressed via the function $P(z)$ of subsection 3.2 .1 as $P(z) / z$.

3.3.5. Symplectic connections on $M^{2 n}$. Similarly, let us count moduli of Fedosov structures $(\omega, \nabla)$, consisting of a symplectic form $\omega$ on $M$ and a linear symmetric connection $\nabla$ such that $\nabla \omega=0$ (note that this condition and $d \omega=0$ imply $T_{\nabla}=0$ [41]). The pairs $(\omega, \nabla)$ with this differential relation form an equation $\mathcal{E}$. In [8] S. Dubrovskiy investigated the number of differential invariants in the "staggered" jet-filtration $j^{k}(\omega, \nabla)=\left(j^{k} \omega, j^{k-1} \nabla\right)$ on $\mathcal{E}$. In contrast, we consider here the natural jet-filtration $j^{k}(\omega, \nabla)=\left(j^{k} \omega, j^{k} \nabla\right)$.

Proposition 9. The orbit dimensions for jets of Fedosov structures are: $h_{0}=0, h_{1}=\frac{1}{2}(n-1) n(2 n+1)(2 n+3)+\delta_{n, 1}, h_{2}=\frac{1}{5} n(n+1)(3 n+$ $2)\left(4 n^{2}-1\right)-\delta_{1, n}$, and $h_{k}=\left(\begin{array}{c}2 n+2 \\ 3\end{array}\right)\left(\begin{array}{c}2 n+k-1 \\ k\end{array}\right)-\left(\begin{array}{c}2 n+k+2 \\ k+3\end{array}\right)$ for $k \geq 3$.

Proof. This structure $(\omega, \nabla)$ is of mixed orders, similarly to the metric case: $r=1$ for $\omega$ and $r=2$ for $\nabla$. It is worth fixing the stabilizer of $\omega$ (since this has the Darboux normal form) to be the (infinitedimensional) pseudo-group of symplectomorphisms $G=\operatorname{Diff}_{\text {loc }}(M, \omega)$, with the Lie algebra sheaf $\mathcal{G}$ whose elements $X_{H}$ are encoded by Hamiltonians defined up to constant terms $H \in C^{\infty}\left(M^{2 n}\right) / \mathbb{R}$.

The condition on the connection to be symplectic means that $\Gamma_{i j k}=$ $\omega_{i a} \Gamma_{j k}^{a}$ is symmetric in all indices in coordinates where $\omega$ is constant [8]. Equivalently, if we fix one symplectic connection $\nabla^{0}$, then any other symplectic connection is $\nabla=\nabla^{0}+A$, where $A \in S^{2} T_{a}^{*} \otimes T_{a}$ is fully symmetric upon $\omega$-lowering the indices: $\omega(A(\cdot, \cdot), \cdot) \in S^{3} T_{a}^{*}$.

Thus, in the reduced form ( $\omega$ fixed, $\nabla$ varies), the geometric structure is given by a section of an affine bundle with the corresponding vector bundle $S^{3} T_{a}^{*}$. Hence the symbols of the equation $\mathcal{E}$ are equal to $g_{k} \simeq$ $S^{k} T_{a}^{*} \otimes S^{3} T_{a}^{*}$. The action of group $G$ has order $r=3$ : the elements depend on 1-jet of the Hamiltonian $H$ and the lift to the space of connections adds two orders.

The stabilizer of a 0-jet is the symplectic group $\mathrm{St}_{a_{0}}^{2}=\operatorname{Sp}\left(T_{a}, \omega\right)$ with the Lie algebra generated by Hessians $d_{a}^{2} H$, and we normalize $H(a)=0$, $d_{a} H=0$. For $n>1$ this stabilizer is resolved on the level of 1 -jets, because the linear symplecic group acts freely on the space of curvatures of $\nabla$ (this space is described in [41]). Thus $h_{0}=\operatorname{dim} g_{0}-\operatorname{dim} \Delta_{3}=0$, $h_{1}=\operatorname{dim} g_{1}-\operatorname{dim} \Delta_{2}-\operatorname{dim} \Delta_{4}$ and $h_{k}=\operatorname{dim} g_{k}-\operatorname{dim} \Delta_{k+3}=0$ for $k \geq 2$ as was claimed.

In the case $n=1$ ( $\operatorname{dim} M=2$ ) the curvature is expressed by the Ricci part and the stabilizer is resolved on the next jet-level, i.e. the action is locally free not from the level of 1 -jet, but from the level of 
2-jets. Again $h_{0}=0$, but now $h_{1}=1$ - the only invariant of order one is the norm of the Ricci tensor of the connection with respect to $\omega$. Thus a 1-dimensional stabilizer exists on this level, but it resolves on the next level, and we have $h_{2}=5, h_{k}=3 k$ for $k \geq 3$. In other words, with respect to the formulae for $n>1$ dimension $h_{1}$ increases by 1 and $h_{2}$ decreases by 1 , the other dimensions being un-changed.

This implies the formula for the Poincaré function:

$$
P(z)= \begin{cases}\frac{z\left(1+3 z-z^{3}\right)}{(1-z)^{2}}, & \text { for } n=1 \\ \frac{2 n\left(2 n^{2}+3 n+1\right) z^{3}-3}{3 z^{3}(1-z)^{2 n}}+\frac{1+2 n z-n(2 n+1) z^{2}\left(z^{2}-1\right)}{z^{3}}, & \text { for } n>1\end{cases}
$$

3.3.6. Projective connections on $M^{n}$. Two linear connections are projectively equivalent if their geodesics coincide as un-parametrized curves. An equivalence class is called a projective connection. Every such structure is represented by a symmetric connection, and two symmetric connections $\nabla, \nabla^{\prime}$ are projectively equivalent iff for some 1-form $\nu$ we have:

$$
\nabla_{X} Y-\nabla_{X}^{\prime} Y=\nu(X) Y+\nu(Y) X
$$

In components, an equivalence class is represented by Thomas' symbols $\Pi_{i j}^{k}=\Gamma_{i j}^{k}-\frac{1}{n+1}\left(\delta_{i}^{k} \Gamma_{a j}^{a}+\delta_{j}^{k} \Gamma_{a i}^{a}\right)$, which is the traceless symmetric part of the Christoffel symbol $\Gamma_{i j}^{k}$.

The number of differential invariants (projective scalars) was computed in [26] (2D projective connections are equivalent to cubic ODE considered by Lie and Tresse, see subsection 3.1.2). We provide an independent short computation.

Proposition 10. For $n>2$ we get $h_{0}=0, h_{1}=\frac{1}{3} n^{2}\left(n^{2}-7\right), h_{2}=$ $\frac{n}{24}(n-2)\left(5 n^{3}+16 n^{2}+15 n+12\right), h_{k}=\frac{n}{2}(n-1)(n+2)\left(\begin{array}{c}n+k-1 \\ k\end{array}\right)-n\left(\begin{array}{c}n+k+1 \\ k+2\end{array}\right)$ for $k>2$. For $n=2, h_{k}=0$ for $k<4$ and $h_{k}=2(k-1)$ for $k \geq 4$.

Proof. These structures are sections of an affine bundle of rank $n\left(\begin{array}{c}n+1 \\ 2\end{array}\right)-$ $n=\frac{1}{2}(n-1) n(n+2)$, whence $\operatorname{dim} g_{k}=\frac{1}{2}(n-1) n(n+2)\left(\begin{array}{c}n+k-1 \\ k\end{array}\right)$.

The symbolic system associated to the action is $\mathfrak{g}_{0}=T_{a}, \mathfrak{g}_{1}=$ $\operatorname{End}\left(T_{a}\right), \mathfrak{g}_{2}=\mathfrak{g}_{1}^{(1)} \simeq T_{a}^{*}$ and $\mathfrak{g}_{i}=0$ for $i>2$. This implies that $\mathrm{St}_{a_{k}}^{k+2}$ stabilize from the level $k=2$, and for generic $a_{2}$ this stabilizer vanishes. Thus the action is locally free from the jet-level 2 , and hence $h_{k}=\operatorname{dim} g_{k}-\operatorname{dim} \Delta_{k+2}$ for $k \geq 3$.

This formula is modified in lower orders as follows: $h_{0}=0$ (no $0^{\text {th }}$ order invariants; increase of $h_{0}$ by $n$ with respect to the general formula), $h_{1}=\operatorname{dim} g_{1}-\operatorname{dim} \Delta_{1}-\operatorname{dim} \Delta_{3}$ ( $1^{\text {st }}$ order invariants are obtained from the curvature tensor through quotient by the group of 
time reparametrizations and the general linear group), $h_{2}=\operatorname{dim} g_{2}-$ $\operatorname{dim} \Delta_{4}+n$ (resolution of the stabilizer from the level of 0 -jets).

This can be also justified by direct rank computation for a system of vector fields [26]. The case $n=2$ is special: the action becomes locally free only starting from the jet-level 3 , and the additional 4-dimensional stabilizer on the level of 1 -jets is resolved on the level of 3-jets.

This computation implies the formula:

$P(z)= \begin{cases}\frac{2 z^{4}(3-2 z)}{(1-z)^{2}}, & \text { for } n=2, \\ \frac{n}{(1-z)^{n}}\left(\left(\begin{array}{c}n+1 \\ 2\end{array}\right)-\frac{1+z^{2}}{z^{2}}\right)-n\left(z^{2}+n z-1-\frac{n z+1}{z^{2}}\right), & \text { for } n>2 .\end{cases}$

3.4. Conformal an related structures on $M^{n}$. A conformal structure is a metric up to re-scaling by a positive function, and so it is a section of the bundle $\left(S^{2} T^{*} M \backslash 0_{M}\right) / \mathbb{R}_{+}$with a non-degenerate representative at every point. For $n=1,2$ all metrics are conformally flat, so to get local invariants we restrict to $n \geq 3$.

3.4.1. General conformal structures. As proven in 25, 19 in the case $n>3, h_{0}=h_{1}=0, h_{2}=\frac{n^{2}\left(n^{2}-1\right)}{12}-n^{2}-1, h_{3}=\frac{1}{24} n\left(n^{4}+\right.$ $\left.2 n^{3}-5 n^{2}-14 n-32\right)$ and $h_{k}=\left(\left(\begin{array}{c}n+1 \\ 2\end{array}\right)-1\right) \cdot\left(\begin{array}{c}n+k-1 \\ k\end{array}\right)-n \cdot\left(\begin{array}{l}n+k \\ k+1\end{array}\right)=$ $\frac{n(k-1)}{2}\left(\begin{array}{c}n+k-1 \\ k+1\end{array}\right)-\left(\begin{array}{c}n+k-1 \\ k\end{array}\right)$ for $k \geq 4$. In the case $n=3, h_{0}=h_{1}=h_{2}=0$, $h_{3}=1, h_{4}=9$ and $h_{k}=k^{2}-4$ for $k \geq 5$. This yields [19]:

$$
P(z)= \begin{cases}\frac{z^{3}(1+z)\left(1+5 z-8 z^{2}+3 z^{3}\right)}{(1-z)^{3}}, & \text { for } n=3, \\
\frac{(n+1) n z-2(n+z)}{2 z(1-z)^{n}}+\frac{n}{z}+\left(1+\left(\begin{array}{l}
n \\
2
\end{array}\right)+n z\right)\left(1-z^{2}\right), & \text { for } n>3 .\end{cases}
$$

3.4.2. Weyl conformal structures on $M^{n}$. A Weyl structure is a pair consisting of a conformal structure $[g]$ and a linear connection $\nabla$ preserving it. In terms of the representative $g$, this means $\nabla g=\omega \otimes g$ for a 1-form $\omega$ on $M$. Conformal re-scaling of the representative $g \mapsto$ $e^{f} g$ results in the shift of Weyl potential $\omega \mapsto \omega+d f$. The resulting equivalence class of pairs $(g, \omega)$ is often considered as a Weyl structure, and we follow this agreement.

Note that $k$-jet of $(g, \omega)$ is equivalent to $k$-jet of $g$ and $(k-1)$-jet of $\nabla$. Indeed, for the Levi-Civita connection of $g$ we have: $\nabla-\nabla^{g}=$ $\omega^{\sharp} \in S^{2} T_{a}^{*} \otimes T_{a}$, where in terms of the Christoffel symbols $\gamma$ of $g$ and $\Gamma$ of $\nabla$, the tensor $\omega^{\sharp}$ is given in coordinates as

$$
\Gamma_{i j}^{k}-\gamma_{i j}^{k}=\left(\omega^{\sharp}\right)_{i j}^{k}=\frac{1}{2}\left(\omega_{i} \delta_{j}^{k}+\omega_{j} \delta_{i}^{k}-g_{i j} \omega^{k}\right) .
$$

However $k$-jet of $\nabla$ yields $(k-1)$-jet of $R_{\nabla}$ and, by taking the skewpart of Ricci, it yields $(k-1)$-jet of $d \omega$; using the freedom in shifting 
the Weyl potential by $d f$, this gives $k$-jet of $\omega$ and hence $k$-jet of $\nabla^{g}$ and (cf. subsection 3.3.3) $(k+1)$-jet of $g$, provided that 0 -jet of $g$ is known.

Thus $k$-jet of the Weyl structure $([g], \nabla)$, for $k>0$, is equivalent to the staggered jet, consisting of $(k+1)$-jet of the metric $g$ and $k$-jet of the Weyl potential $\omega$ modulo the equivalence $(g, \omega) \simeq\left(e^{f} g, \omega+d f\right)$. This is the filtration we will be using in our pseudogroup orbit study. Note that the action of $G=\operatorname{Diff}_{\text {loc }}(M)$ on $([g], \nabla)$ has order $r=1$ in the first component and order $r=2$ in the second component.

Proposition 11. We have: $h_{0}=0, h_{1}=\frac{1}{12}\left(n^{2}-4\right)\left(n^{2}+3\right)+\delta_{2, n}$, $h_{2}=\frac{1}{24} n\left(n^{2}-1\right)\left(n^{2}+2 n+8\right)-\delta_{2, n}$, and $h_{k}=\left(\left(\begin{array}{c}n+1 \\ 2\end{array}\right)-1\right)\left(\begin{array}{l}n+k \\ k+1\end{array}\right)+$ $n\left(\begin{array}{c}n+k-1 \\ k\end{array}\right)-n\left(\begin{array}{c}n+k+1 \\ k+2\end{array}\right)$ for $k>2$.

Proof. It is easy to see that the group $\Delta_{1} \oplus \Delta_{2}$ acts transitively on the 0jets $([g], \Gamma)$. The stabilizer is $\mathrm{St}_{a_{0}}^{2}=C O(n) \subset \Delta_{1}$. The action of $\Delta_{3}$ on 1-jet of $\nabla \equiv \Gamma$ is free and the previous stabilizer $C O(n)$ is resolved upon the action on the space of curvature tensors $\left\{R_{\nabla}\right\}$, so that $\mathrm{St}_{a_{1}}^{3}=0$ for $n>2$; in the case $n=2$ the scalar part of $C O(2)=\mathbb{R}^{*} \times S O(2)$ is reduced to $\mathbb{Z}_{2}$, while the rotation persists: $\mathrm{St}_{a_{1}}^{3}=O(2)$; both are resolved on the next jet-level: $\mathrm{St}_{a_{2}}^{4}=0$.

Thus the action is (locally) free from the level of 1-jets for $n>2$ and 2-jets for $n=2$. This allows computing the counting function $h_{k}$. Indeed, the symbol space $g^{\prime}$ for conformal structures satisfies $\operatorname{dim} g_{k}^{\prime}=\left(\left(\begin{array}{c}n+1 \\ 2\end{array}\right)-1\right)\left(\begin{array}{c}n+k-1 \\ k\end{array}\right)$, and the symbol space $g^{\prime \prime}$ for Weyl potentials (the scaling factor $f$ is taken into consideration when counting $g_{k}^{\prime}$ ) satisfies $\operatorname{dim} g_{k}^{\prime \prime}=n\left(\begin{array}{c}n+k-1 \\ k\end{array}\right)$. Thus for $k=1$ we compute $h_{1}=\operatorname{dim} g_{2}^{\prime}+$ $\operatorname{dim} g_{1}^{\prime \prime}-\operatorname{dim} \Delta_{3}-\operatorname{dim} C O(n)$; note also that this equals the dimension of the space of the curvature tensors of $\nabla$ (counting also the skew-part of Ricci) mod stabilizer group action: $h_{1}=\frac{n^{2}\left(n^{2}-1\right)}{12}+\left(\begin{array}{l}n \\ 2\end{array}\right)-\operatorname{dim} C O(n)=$ $\frac{1}{12}\left(n^{2}-4\right)\left(n^{2}+3\right)$. For $k>1$ we get $h_{k}=\operatorname{dim} g_{k+1}^{\prime}+\operatorname{dim} g_{k}^{\prime \prime}-\operatorname{dim} \Delta_{k+2}$. In the case $n=2$ the number $h_{1}$ shall be increased by 1 and $h_{2}$ decreased by 1 .

This computation yields for $n>1$ the formula:

$P(z)=\frac{n z^{2}+\left(\left(\begin{array}{c}n+1 \\ 2\end{array}\right)-1\right) z-n}{z^{2}(1-z)^{n}}-\frac{\left(\left(\begin{array}{l}n \\ 2\end{array}\right)+1\right) z\left(z^{2}-1\right)-n}{z^{2}}-\delta_{2, n} z(z-1)$.

3.4.3. Einstein-Weyl structures on $M^{n}$. The Einstein-Weyl condition is the following set of $\left(\begin{array}{c}n+1 \\ 2\end{array}\right)-1$ equations $\operatorname{Ric}_{\nabla}^{\text {sym }}=\Lambda g$, where $\Lambda=\frac{1}{n} \operatorname{Tr}_{g} \operatorname{Ric}_{\nabla}^{\mathrm{sym}}$, on the unknown Weyl structure $[(g, \omega)]$. This condition is vacuous for $n=2$, so we assume $n>2$ for this structure. 
Proposition 12. We have: $h_{0}=0, h_{1}=\frac{1}{12}(n-3) n(n+1)(n+2)+\delta_{n, 3}$, $h_{2}=\frac{1}{24} n(n-1)(n-2)\left(n^{2}+5 n+8\right)-\delta_{n, 3}$, and $h_{k}=\left(\left(\begin{array}{c}n+1 \\ 2\end{array}\right)-1\right)\left(\begin{array}{l}n+k \\ k+1\end{array}\right)+$ $n\left(\begin{array}{c}n+k-1 \\ k\end{array}\right)-\left(\left(\begin{array}{c}n+1 \\ 2\end{array}\right)-1\right)\left(\begin{array}{c}n+k-2 \\ k-1\end{array}\right)-n\left(\begin{array}{c}n+k+1 \\ k+2\end{array}\right)$ for $k>2$.

Proof. There are two important specifications in this case. First, the structures are given by a differential system $\mathcal{E}$ on $[(g, \omega)]$. It consists of $\left(\begin{array}{c}n+1 \\ 2\end{array}\right)-1$ equations of the second order. This system is determined (not as it stands, because it has more dependent variables $\left(\begin{array}{c}n+1 \\ 2\end{array}\right)+n-1$ than the equations, but determinacy comes modulo the diffeomorphism freedom; see [10] for an effective quotient in the arguably most important case $n=3)$, so its prolongation will have $r_{k}=\left(\left(\begin{array}{c}n+1 \\ 2\end{array}\right)-1\right)\left(\begin{array}{c}n+k-1 \\ k\end{array}\right)$ equations of order $k+2$ on $g$.

Second, we still have $\mathrm{St}_{a_{0}}^{2}=C O(n)$ but the stabilizer $\mathrm{St}_{a_{1}}^{3}$ changes. While for $n>3$ the curvature of $\nabla$ contains the Weyl tensor as an irreducible part (reducible into anti- and self-dual parts for $n=4$ ) and the action of $C O(n)$ resolves on it, in the case $n=3$ the curvature of $\nabla$ consists of the trace part of $\mathrm{Ric}_{\nabla}^{\mathrm{sym}}$ (due to Einstein-Weyl condition) and $\operatorname{Ric}_{\nabla}^{\text {skew }} \equiv d \omega$ thus reducing $\mathrm{St}_{a_{0}}^{2}=C O(3)$ to $\mathrm{St}_{a_{1}}^{3}=O(2)$, and in the next jet-level this stabilizer is also resolved. Thus we conclude $\operatorname{dim} \mathrm{St}_{a_{1}}^{3}=\delta_{n, 3}$ and $\operatorname{dim} \mathrm{St}_{a_{k}}^{k+2}=0$ for $k \geq 2$.

These two observations imply: $h_{1}=\frac{n^{2}\left(n^{2}-1\right)}{12}+\left(\begin{array}{c}n \\ 2\end{array}\right)-r_{0}-\operatorname{dim} C O(n)=$ $\frac{1}{12}(n-3) n(n+1)(n+2)$. For $k>1$ we get: $h_{k}=\operatorname{dim} g_{k+1}^{\prime}+\operatorname{dim} g_{k}^{\prime \prime}-$ $r_{k-1}-\operatorname{dim} \Delta_{k+2}$. In the case $n=3$ the number $h_{1}$ shall be increased by 1 and $h_{2}$ decreased by 1 , implying the claim.

This computation yields for $n>2$ the formula:

$$
P(z)= \begin{cases}\frac{z\left(1+5 z-z^{2}-z^{3}\right)}{(1-z)^{2}}, & \text { for } n=3, \\
\frac{n z^{2}-\left(\left(\begin{array}{c}
n+1 \\
2
\end{array}\right)-1\right) z\left(z^{2}-1\right)-n}{z^{2}(1-z)^{n}}-\frac{\left.\left(\begin{array}{c}
n \\
2
\end{array}\right)+1\right) z\left(z^{2}-1\right)-n}{z^{2}}, & \text { for } n>3 .\end{cases}
$$

Remark. For $n=3$ computation of the Poincaré function in a different jet-filtration for Weyl and Einstein-Weyl structures was done recently in [21]. The results differ from the above, but agree in asymptotic. This is an effect of "staggering" jets or "normalizing" the structure.

3.4.4. Self-dual conformal structures in 4D. Self-duality equation $* W_{g}=W_{g}$ for the Weyl tensor of metric $g$ (of Riemannian or neutral signature) has meaning only in dimension 4 . The count for differential invariants for self-dual conformal structures $[g]$ on $M^{4}$ was performed in [20]: $h_{0}=h_{1}=0, h_{2}=1, h_{3}=13, h_{k}=3 k^{2}-7$ for $k>3$ (note the difference with subsection 3.2 .3 , where we considered $g$ intead of $[g]$ ). 
Consequently the Poincaré function is [20]:

$$
P(z)=\frac{z^{2}\left(1+10 z+5 z^{2}-17 z^{3}+7 z^{4}\right)}{(1-z)^{3}} .
$$

3.5. Almost complex structures on $M^{2 n}$. This structure of order $r=1$ is given by a field $J \in \operatorname{End}(T M)$ with $J^{2}=-\mathbf{1}$. This is the first non-trivial example of an infinite type geometric structure, meaning that its symbol allows infinite-dimensional symmetry algebra, which is realized for the standard (integrable) complex structure on $\mathbb{C}^{n}$, though generic almost complex structures have no local symmetry at all [15].

In more details, $J$ is a $G$-structure with the group $G=\operatorname{GL}(n, \mathbb{C})$, whose Lie algebra $\mathfrak{g}=\mathfrak{g l}(n, \mathbb{C})$ has infinite type: its prolongation is the algebra $S\left(\mathbb{C}^{n}\right)^{*} \otimes_{\mathbb{C}} \mathbb{C}^{n}$ of formal holomorphic vector fields at 0 . However the prolongation-projection of the Lie equation for $J$ encodes conservation of both $J$ and its Nijenhuis tensor $N_{J}$. For $n>2$ this is already a finite type structure in general. But for $n=2$ it is still of infinite type, and one has to do one more prolongation-projection to achieve finite type.

Theorem 13. For almost complex structure the count of invariants is as follows: $h_{0}=0$ and $h_{k}=2 n^{2}\left(\begin{array}{c}2 n+k-1 \\ k\end{array}\right)-2 n\left(\begin{array}{c}2 n+k \\ k+1\end{array}\right)+2 n\left(\begin{array}{c}n+k \\ k+1\end{array}\right)-$ $2 n\left(\begin{array}{c}n+k-1 \\ k\end{array}\right)+2\left(\delta_{k, 1}-\delta_{k, 2}\right) \delta_{n, 3}$, for $k>0, n \geq 3$. In the case $n=2$, $h_{0}=h_{1}=0, h_{2}=2$ and $h_{k}=8\left(\begin{array}{c}k+3 \\ k\end{array}\right)-4\left(\begin{array}{c}k+4 \\ k+1\end{array}\right)+4$.

Proof. We will do this computation in several steps.

First note that $\mathrm{St}_{a_{0}}^{1} \simeq G=\operatorname{GL}(n, \mathbb{C})$. Since $J$ is a $G$-structure with $\mathfrak{g}=\operatorname{Lie}(G) \subset \operatorname{End}\left(T_{a}\right)$, Proposition 4 shows that $\mathrm{St}_{a_{k}}^{k+1} \supset \mathfrak{g}_{k+1}=\mathfrak{g}^{(k)}=$ $S_{\mathbb{C}}^{k+1} T_{a}^{*} \otimes_{\mathbb{C}} T_{a}$. The latter space is

$\left\{\Phi: S^{k+1} T_{a} \rightarrow T_{a}, \Phi\left(X_{0}, \ldots, J X_{i}, \ldots, X_{k}\right)=J \Phi\left(X_{0}, \ldots, X_{i}, \ldots, X_{k}\right)\right\}$

and has (real) dimension $2 n\left(\begin{array}{c}n+k \\ k+1\end{array}\right)$. The analog of freeness from the jet-level $k$ is the equality in the previous inclusion.

The equation for symmetries of $J$ has in 1-prolongation the condition that $N_{J}$ is preserved. Consequently, the corresponding symbolic system

$$
\tilde{\mathfrak{g}}=\left\{\Phi: T_{a} \rightarrow T_{a}: J \Phi=\Phi J, N_{J}(\Phi \cdot, \cdot)+N_{J}(\cdot, \Phi \cdot)=\Phi N_{J}(\cdot, \cdot)\right\}
$$

is of finite type for generic $N_{J}$ and $n \geq 3$. Indeed, consider the bundle $\tilde{\mathcal{E}}$ consisting of the pair $J$ and its Nijenhuis tensor $N_{J}$, namely $\tilde{a}_{0}=\left(J, N_{J}\right)$. The tensor $N_{J}$ involves 1 -jet of $J$, yet the action of the pseudogroup $\operatorname{Diff}$ loc $(M)$ on $\tilde{\mathcal{E}}$ still has order $r=1$. Let ${\widetilde{\mathrm{St}^{a}}}_{\tilde{a}_{k}}^{k+1} \subset \mathbb{D}_{a}^{k+1}$ denote the corresponding stabilizers. 
Lemma 14. For $n>3$ and generic $\tilde{a}_{0}$ (i.e. generic 1-jet of $J$ ) we have:

$$
\widetilde{\mathrm{St}}_{\tilde{a}_{0}}^{1}=\left\{\Phi \in \operatorname{End}\left(T_{a}, J\right): N_{J}(\Phi \cdot, \Phi \cdot)=\Phi N_{J}(\cdot, \cdot)\right\}=\mathbb{Z}_{3}
$$

Proof. Identifying $\left(T_{a}, J\right)$ with $\mathbb{C}^{n}$ observe that scaling by $e^{2 \pi i / 3}$ always belongs to $\widetilde{\mathrm{St}}_{\tilde{a}_{0}}^{2}$. To show that generically there are no other symmetries, note that the subgroup ${\widetilde{\mathrm{St}^{2}}}_{\tilde{a}_{0}}^{2} \subset \mathrm{GL}(n, \mathbb{C})$ is upper-semicontinuous in $N_{J} \in \Lambda^{2}\left(\mathbb{C}^{n}\right)^{*} \otimes_{\overline{\mathbb{C}}} \mathbb{C}^{n}$, so if we show the claim for one $N_{J}$ it will follow for a Zariski generic element as well.

Consider the following element given in terms of a complex basis $e_{1}, \ldots, e_{n} \in \mathbb{C}^{n}$ :

$$
\begin{aligned}
& N_{J}\left(e_{1}, e_{k}\right)=e_{k+1}(2 \leq k<n), N_{J}\left(e_{1}, e_{n}\right)=e_{2}, \\
& N_{J}\left(e_{2}, e_{3}\right)=e_{1}, N_{J}\left(e_{2}, e_{k}\right)=k e_{k}(3<k \leq n) .
\end{aligned}
$$

A moment of thought shows that the only complex transformations preseving this $N_{J}$ are diagonal, i.e. $\Phi\left(e_{k}\right)=\rho_{k} e^{i \theta_{k}} e_{k}$, where $\rho_{k} \in \mathbb{R}_{+}$ and $\theta_{k} \in \mathbb{R} \bmod 2 \pi$. The first line of the defining relations yields $\rho_{1}=1$, $\rho_{2}=\cdots=\rho_{n}$, and then we get that all $\rho_{k}=1$. After this it is easy to obtain $\theta_{k}=\theta \in\{0,2 \pi / 3,4 \pi / 3\}$.

Thus, for $n>3$ we have $\tilde{\mathfrak{g}}_{1}=0$, and hence $\tilde{\mathfrak{g}}_{k}=0$ for $k>1$.

For $n=3$ the normal forms of [15] yield $\operatorname{dim} \tilde{\mathfrak{g}}_{1}=2$ for generic $N_{J}$ and a straightforward computation shows that $\tilde{\mathfrak{g}}_{2}=0$.

Proposition 15. In the case $n>3$ we have $\mathrm{St}_{a_{k-1}}^{k}=\mathfrak{g}_{k}$ for $k>1$ and generic $a_{k-1} \in \mathcal{E}^{k-1}$. When $n=3$ this equality holds true as well except for $k=2$, in which case we have $\operatorname{dim} \mathrm{St}_{a_{1}}^{2} / \mathfrak{g}_{2}=2$ for generic $a_{1} \in \mathcal{E}^{1}$.

Proof. If a diffeomorphism $\varphi$ preserves the $k$-jet of $J$, then it preserves $(k-1)$-jet of $\left(J, N_{J}\right)$. Hence an injective map $\mathrm{St}_{a_{k}}^{k+1} / \mathfrak{g}_{k+1} \rightarrow{\widetilde{\mathrm{St}^{a_{k-1}}}}_{\tilde{a}_{k}}^{k}$ (in fact, an isomorphism). These can be united into a commutative diagram

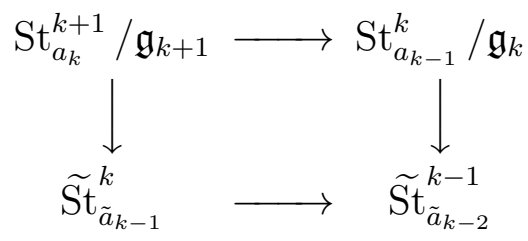

By Proposition 4 the kernel of the bottom map is ${\widetilde{\mathrm{St}^{a}}}_{\tilde{a}_{k-1}}^{k} \cap \Delta_{k}=\tilde{\mathfrak{g}}_{k}$, and so by induction $\widetilde{\mathrm{St}}_{\tilde{a}_{k-1}}^{k}=0$ for $n>3$, implying the first claim. 
For $n=3$ the upper arrow of the diagram is injective. Moreover it can be directly checked (for instance, via the normal forms of [15]) that $\widetilde{\mathrm{St}}_{\tilde{a}_{1}}^{2}=0$, and so $\mathrm{St}_{a_{k}}^{k+1}=\mathfrak{g}_{k+1}$ for $k \geq 2$. The same equality fails for $k=1$. A straightforward computation in Maple gives $\operatorname{dim} \mathrm{St}_{a_{1}}^{2}=38$, while $\operatorname{dim} \mathfrak{g}_{2}=36$, implying the second claim.

The space of all almost complex structures $\mathcal{E}$ is the fiber bundle with fiber $F=G L(2 n, \mathbb{R}) / G L(n, \mathbb{C})$ of dimension $2 n^{2}$, and the $k$-symbol is $g_{k}=S^{k} T^{*} M \otimes T F$. Consequently, the number of pure order $k>0$ differential invariants for $n>3$ is (for $k=0$ we have $h_{0}=0$ ):

$$
\begin{aligned}
& h_{k}=\operatorname{dim} g_{k}-\operatorname{dim} \Delta_{k+1}+\operatorname{dim} \mathfrak{g}_{k+1}-\operatorname{dim} \mathfrak{g}_{k} \\
= & 2 n^{2}\left(\begin{array}{c}
2 n+k-1 \\
k
\end{array}\right)-2 n\left(\begin{array}{c}
2 n+k \\
k+1
\end{array}\right)+2 n\left(\begin{array}{c}
n+k \\
k+1
\end{array}\right)-2 n\left(\begin{array}{c}
n+k-1 \\
k
\end{array}\right) .
\end{aligned}
$$

For $n=3$ we have modification $h_{1}=2, h_{2}=64$, i.e. $h_{k} \mapsto h_{k}+2\left(\delta_{k, 1}-\right.$ $\left.\delta_{k, 2}\right) \delta_{n, 3}$ due to existence of 2 scalar invariants of order 2 [15].

In the case $n=2$ the situation is more complicated: $N_{J}$ is encoded by $J$-invariant subspace ( $\mathbb{C}$-line) $\Pi_{a}=\operatorname{Im} N_{J} \subset T_{a}$ and an element of a $\mathbb{C}$-line $\left(T_{a} / \Pi_{a}\right)^{*} \otimes_{\mathbb{C}} \operatorname{End}_{\overline{\mathbb{C}}}\left(\Pi_{a}\right)$. Thus the system $\tilde{\mathfrak{g}}$, obtained by prolongation-projection is not of finite type: $\tilde{\mathfrak{g}}_{k+1}=\tilde{\mathfrak{g}}^{(k)}$ satisfies $\operatorname{dim} \tilde{\mathfrak{g}}_{k+1}=2$ for all $k>0$, while $\operatorname{dim} \tilde{\mathfrak{g}}_{1}=4$. Indeed, we have in a complex basis $X_{1} \in \Pi_{a}, X_{2} \in T_{a} \backslash \Pi_{a}$ of $T_{a}$ :

$$
\tilde{\mathfrak{g}}=\left\{\Phi:\left(T_{a}, J\right) \rightarrow\left(T_{a}, J\right) \mid \Phi=\left(\begin{array}{cc}
\rho-i \theta & b \\
0 & 2 i \theta
\end{array}\right), \rho, \theta \in \mathbb{R}, b \in \mathbb{C}\right\} .
$$

Therefore the prolongation $\tilde{\mathfrak{g}}_{k} \subset S_{\mathbb{C}}^{k} T_{a}^{*} \otimes_{\mathbb{C}} T_{a}$ consists of elements $\Phi_{k}$ with $i_{v} \Phi_{k}=0 \forall v \in \Pi_{a}$ and $\operatorname{Im} \Phi_{k} \in \Pi_{a}$. In other words, for $k>1$ $\Phi_{k} \in S_{\mathbb{C}}^{k}\left(T_{a} / \Pi_{a}\right) \otimes_{\mathbb{C}} \Pi_{a}$ and the latter space has real dimension 2 .

The next prolongation-projection is encoded by a complete parallelism, namely the points of $\underset{\tilde{\mathcal{E}}}{\tilde{C}}$ are frames related to $\left(J, N_{J},[\Pi, \Pi]\right)[15$ (so determined by the 2-jet of $J$ ), whence $\tilde{\tilde{\mathfrak{g}}}_{k}=0$ for $k>0$.

Proposition 16. For $n=2$ and $k \geq 3$ the map $\mathrm{St}_{a_{k}}^{k+1} / \mathfrak{g}_{k+1} \rightarrow$ $\mathrm{St}_{a_{k-1}}^{k} / \mathfrak{g}_{k}$ is an isomorphism and the spaces have dimensions 2.

Proof. By the argument from the proof of Proposition 15 we have an injective map $\widetilde{\mathrm{St}}_{\tilde{a}_{k}}^{k+1} / \tilde{\mathfrak{g}}_{k+1} \rightarrow \widetilde{\mathrm{St}}_{\tilde{a}_{k-1}}^{k} / \tilde{\mathfrak{g}}_{k}$ for $k>0$ and moreover the source spaces vanish implying $\widetilde{\mathrm{St}}_{\tilde{a}_{k-1}}^{k}=\tilde{\mathfrak{g}}_{k}$ for $k>1$. Now we can use the commutative diagram from the proof of Proposition 15 again. It implies that $\mathrm{St}_{a_{k}}^{k+1}$ contains both $\mathfrak{g}_{k+1}$ and $\tilde{\mathfrak{g}}_{k}$, and the stabilization means that nothing more contributes. 
We conclude that the parts $\mathfrak{g}_{k}, \tilde{\mathfrak{g}}_{k-1}$ of the stabilizer $\mathrm{St}_{a_{k-1}}^{k}$ resolve upon prolongation to $k$-jets $\mathcal{E}^{k}$, but in the new stabilizer $\mathrm{St}_{a_{k}}^{k+1}$ the parts $\mathfrak{g}_{k+1}, \tilde{\mathfrak{g}}_{k}$ appear instead. This proves the claim.

For a generic $a_{\infty}=\left\{a_{k}\right\} \in \mathcal{E}$ the sequence $\left\{\operatorname{dim} \mathrm{St}_{a_{k}}^{k+1}\right\}_{k=0}^{\infty}$ is equal to $\{8,16,18,22,26,30, \ldots\}$ by a straightforward (albeit very demanding) Maple computation. Its grows stabilizes starting from $k=3$ in accordance with Proposition 16. This implies the following dimension formulae for $n=2$ and $k>2$ :

$$
\begin{aligned}
& h_{k}=\operatorname{dim} g_{k}- \operatorname{dim} \Delta_{k+1}+\operatorname{dim} \mathrm{St}_{a_{k}}^{k+1}-\operatorname{dim} \mathrm{St}_{a_{k-1}}^{k} \\
&=\operatorname{dim} g_{k}- \operatorname{dim} \Delta_{k+1}+\operatorname{dim} \mathfrak{g}_{k+1}-\operatorname{dim} \mathfrak{g}_{k}+\operatorname{dim} \tilde{\mathfrak{g}}_{k}-\operatorname{dim} \tilde{\mathfrak{g}}_{k-1} \\
&=8\left(\begin{array}{c}
k+3 \\
k
\end{array}\right)-4\left(\begin{array}{c}
k+4 \\
k+1
\end{array}\right)+4=\frac{2}{3} k^{3}+2 k^{2}-\frac{8}{3} k-4 .
\end{aligned}
$$

For $k \leq 2$ we have: $h_{0}=h_{1}=0$, but $h_{2}=2$ (as a straightforward Maple computation verifies). This finishes proof of the theorem.

Let us list the numbers of the pure order $k$ invariants for the first $n$ :

\begin{tabular}{c||c|c|c|c|c|c|c|l} 
& $h_{0}$ & $h_{1}$ & $h_{2}$ & $h_{3}$ & $h_{4}$ & $h_{5}$ & $h_{6}$ & $\ldots$ \\
\hline$n=2$ & 0 & 0 & 2 & 24 & 60 & 116 & 196 & $\ldots$ \\
\hline$n=3$ & 0 & 2 & 64 & 282 & 792 & 1806 & 3612 & $\ldots$ \\
\hline$n=4$ & 0 & 16 & 272 & 1320 & 4392 & 11840 & 27744 & $\ldots$
\end{tabular}

The formulae of the theorem are encoded via the Poincaré function:

$$
P(z)= \begin{cases}\frac{2 z^{2}\left(1+8 z-12 z^{2}+6 z^{3}-z^{4}\right)}{(1-z)^{4}}, & \text { for } n=2, \\ \frac{2 z\left(1+26 z-36 z^{2}+10 z^{3}+17 z^{4}-18 z^{5}+7 z^{6}-z^{7}\right)}{(1-z)^{6}}, & \text { for } n=3, \\ \frac{2 n(n z-1)}{z(1-z)^{2 n}}+\frac{2 n}{z(1-z)^{n-1}}+2 n, & \text { for } n>3 .\end{cases}
$$

\section{Conclusion: Towards the General Arnold Conjecture}

By the Hilbert-Serre theorem, the Poincaré series of a finitely generated graded module over an algebra with homogeneous generators of degrees $d_{1}, \ldots, d_{n}$ has the form (see [36], also for many examples)

$$
P(z)=\frac{F(z)}{\prod_{i=1}^{n}\left(1-z^{d_{i}}\right)} .
$$

This is more general than the one given by formula (2). Indeed, the poles are on the unit circle $S^{1} \subset \mathbb{C}$, but can be other roots of unity.

More general Poincaré functions arise in the problems of analysis when the pseudogroup $G$ acts non-transitively on the base. This is the 
case in singularity theory. For instance, the pseudogroup of symplectomorphisms $G=\operatorname{Diff}_{\text {loc }}\left(\mathbb{R}^{2 n}, \omega\right)$ acting on the space of germs of critical linearly stable Hamiltonians ( $\mathrm{Sp} \equiv$ spectrum)

$$
\mathcal{E}=\left\{H \in C_{\mathrm{loc}}^{\infty}\left(\mathbb{R}^{2 n}, 0\right): H(0)=0, d_{0} H=0, \operatorname{Sp}\left(\omega^{-1} d_{0}^{2} H\right) \subset i \mathbb{R}\right\}
$$

was considered in [23]: the corresponding Poincaré function on the general stratum has multiple poles at \pm 1 :

$$
P(z)=\frac{1}{\left(1-z^{2}\right)^{n}} .
$$

Another classical problem is related to the Poincaré-Dulac normal form for a vector field $v$ near stationary point $0 \in \mathbb{R}^{n}(x), v(0)=0$. Let $d_{0} v$ have spectrum $\Lambda=\left(\lambda_{1}, \ldots, \lambda_{n}\right)$. Then $v$ is formally equivalent to a vector field $w$ with components (no summation by $i$ )

$$
w^{i}=\lambda^{i} x_{i}+\sum_{m \in R_{i}(\Lambda)} c_{m}^{i} x^{m}
$$

where $R_{i}(\Lambda)=\left\{m \in\left(\mathbb{Z}_{\geq 0}\right)^{n}: m_{i}=\langle m, \lambda\rangle,|m|=\sum m_{i} \geq 2\right\}$ is the $i$ th resonance set and $x^{m}=x_{1}^{m_{1}} \cdots x_{n}^{m_{n}}$, see [2]. Some of the coefficients can be further normalized leaving only the essential ones.

Clearly, $\operatorname{Sp}\left(d_{0} v\right)=\operatorname{Sp}\left(d_{0} w\right)=\Lambda$ and the normalized coefficients $c_{m}^{i}$ are differential invariants. The corresponding counting function $P(z)$ is rational in all known cases, but it is not arbitrary. To see this consider the case $n=2$. Here are the main singularities:

(1) Non-resonant case: formal linearization, whence

$$
P(z)=2 z
$$

(2) $\lambda_{1} / \lambda_{2}=m$ (or $\frac{1}{m}$ ) with $m \in \mathbb{N}$ fixed, $m>1$ (Poincaré domain), then there is only one non-resonant term [2] and

$$
P(z)=z+z^{m}
$$

(3) $\lambda_{1} / \lambda_{2} \in \mathbb{Q}$ - fixed (Siegel domain: here $\lambda_{1}, \lambda_{2} \in \mathbb{R} \cup i \mathbb{R}$ ), there is infinity of non-resonant terms, but the normal form leaves only few of them. A general saddle resonant singularity has the normal form $(p, q \in \mathbb{N}$ are fixed $) v=p \lambda\left(x+x^{q+1} y^{p}\right) \partial_{x}-q \lambda(y+$ $\left.a x^{q} y^{p+1}+b x^{2 q} y^{2 p+1}\right) \partial_{y}$ [42] (an elliptic singularity has a similar normal form). Thus $h_{1}=h_{m+1}=h_{2 m+1}=1$ for $m=p+q$ and $h_{i}=0$ else, implying

$$
P(z)=z+z^{m+1}+z^{2 m+1}
$$


(4) $\lambda_{1} \neq 0, \lambda_{2}=0$ (or otherwise around) - the saddle-node point; the normal form here is $v=\lambda\left(x Q_{m}(x) \partial_{x} \pm\left(y^{m+1}+a y^{2 m+1}\right) \partial_{y}\right)$, where $Q_{m}(x)$ is a polynomial of $\operatorname{deg}=m$ [14] and consequently

$$
P(z)=\frac{z-z^{m+1}}{1-z}+z^{2 m+1}
$$

(5) $\lambda_{1}=\lambda_{2}=0$, but $d_{0} v \neq 0$ (nilpotent linear part), this is the Takens-Bogdanov singularity; the pre-normal Lienard form is $v=y \partial_{x}+x(x a(x)+y b(x)) \partial_{y}$. It can be checked that for $a(0) \neq$ $0 \neq b(0)$ a formal change of variables yields $b(x)=0, a(x)=$ $\sum_{k \in \Delta} a_{k} x^{k}$, where $\Delta=\left\{n \in \mathbb{Z}_{\geq 0}: n \notin 3 \mathbb{N}+1\right\}$; alternatively one can eliminate $a(x)$ except for two first terms and one thrid of the terms of $b(x)$ [43]. Both normal forms imply that $h_{k}$ is the characteristic function $\chi_{\Delta}(k)$, whence

$$
P(z)=\frac{\left(1+z+z^{2}-z^{4}\right) z^{2}}{1-z^{3}} .
$$

Further normal forms for more complicated degenerations can be found in [37, they lead to other rational Poincaré functions.

The mechanism explaining this rationality is not the same as in the Lie-Tresse theorem, see the discussion in [23]. In Section 1 we derived the strong form of Arnold's conjecture provided the pseudogroup $G$ acts transitively on $M$, and we showed many explicit computations in Section 3. It seems plausible that this approach can be extended to the case when $G$-orbits foliate $M$. However, in the presence of singular orbits, the general Arnold conjecture is still wide open.

\section{REFERENCES}

[1] V.I. Arnold, Mathematical problems in classical physics, Trends and perspectives in applied mathematics, Appl. Math. Sci. 100, 120, Springer-Verlag (1994); also: Arnolds problems, Fazis, Moscow (2000); Springer, Berlin (2004).

[2] V.I. Arnold, "Additional chapters of the theory of ordinary differential equations", Moscow, Nauka (1978) [Russian]; Engl. transl. "Geometrical methods in the theory of ordinary differential equations", Springer-Verlag (1988).

[3] A. Besse, Einstein manifolds, Springer-Verlag, Berlin Heidelberg (1987).

[4] P. Bibikov, On Lies problem and differential invariants of ODEs $y^{\prime \prime}=F(x, y)$, Funct. Anal. Appl. 51:4, 16-25 [Engl.transl: 255-262] (2017).

[5] M. V. Babich, L. A. Bordag, Projective differential geometrical structure of the Painlevé equations, J. Differ. Equations 157, no.2, 452-485 (1999).

[6] E. Cartan, Sur les variétés à connexion projective, Bull. Soc. Math. France 52, 205-241 (1924).

[7] S. Dubrovskiy, Moduli space of symmetric connections, Zap. Nauchn. Sem. SPb. otd. Steklov Inst. Math. 292 (2002); J. Math. Sci. (N.Y.) 126, no.2, 1053-1063 (2005). 
[8] S. Dubrovskiy, Moduli space of Fedosov structures, Ann. Glob. Anal. Geom. 27, 273-297 (2005).

[9] S. Dubrovskiy, Moduli space of general connections, arXiv:1010.5285 (2010).

[10] M. Dunajski, E.V. Ferapontov, B. Kruglikov, On the Einstein-Weyl and conformal self-duality equations, J. Math. Phys. 56, 083501 (2015).

[11] A. Gordillo, J. Navarro, On Moduli Spaces for Finite-Order Jets of Linear Connections, Filomat 31:7, 2035204 (2017).

[12] C.N. Haskins, On the invariants of quadratic differential forms, Transactions Amer. Math. Soc. 3, 71-91 (1902).

[13] D. Hilbert, Theory of algebraic invariants (translated from the German original), Cambridge University Press, Cambridge (1993).

[14] Y. Ilyashenko, S. Yakovenko, Lectures on analytic differential equations, Graduate Studies in Mathematics 86, Amer. Math. Soc. (2008).

[15] B. Kruglikov, Non-existence of higher-dimensional pseudoholomorphic submanifolds, Manuscripta Mathematica, 111 (2003), 51-69.

[16] B. Kruglikov, Point Classification of Second Order ODEs: Tresse Classification Revisited and Beyond, Abel Symposia 5, 199-221, Springer (2009).

[17] B. Kruglikov, Involutivity of the field equations, Journ. Math. Phys. 51, 032502 (2010).

[18] B. Kruglikov, Differential Invariants and Symmetry: Riemannian Metrics and Beyond, Lobachevskii Journal of Mathematics 36, no.3, 292-297 (2015).

[19] B. Kruglikov, Conformal Differential Invariants, Journal of Geometry and Physics 113, 170175 (2017).

[20] B. Kruglikov, E. Schneider, Differential invariants of self-dual conformal structures, Journal of Geometry and Physics 113, 176187 (2017).

[21] B. Kruglikov, E. Schneider, Differential invariants of Einstein-Weyl structures in 3D, arXiv:1802.00702 (2018).

[22] B. Kruglikov, V. Lychagin, Geometry of Differential equations, in: Handbook of Global Analysis, Ed. D.Krupka, D.Saunders, Elsevier, 725-772 (2008).

[23] B. Kruglikov, V. Lychagin, Global Lie-Tresse theorem, Selecta Math. 22, 13571411 (2016).

[24] A. Kumpera, Invariants differentiels d'un pseudogroupe de Lie. I-II. J. Differential Geometry 10, no. 2, 289-345; no. 3, 347-416 (1975).

[25] J. Levine, Conformal scalars, Bull.AMS 42, no.2, 115-124 (1936).

[26] J. Levine, Projective scalar differential invariants, Ann. Math. 37, no.3, 618634 (1936).

[27] S. Lie, Klassifikation und Integration von gewöhnlichen Differentialgleichungen zwischen $x, y$, die eine Gruppe von Transformationen gestatten. III, Archiv for Mathematik og Naturvidenskab 8 (Kristiania, 1883), 371-458; Gesam. Abh. Bd. 5 (1924), paper XIV, 362-427.

[28] S. Lie, Ueber Differentialinvarianten, Math. Ann. 24, no. 4, 537-578 (1884).

[29] R. Liouville, Sur les invariants de certaines équations différentielles et sur leurs applications, Journal de l'École Polytechnique 59 (1889), 7-76.

[30] V. Lychagin, V. Yumaguzhin, Invariants in Relativity Theory, Lobachevskii Journal of Mathematics 36, no.3, 298-312 (2015).

[OP] P. Olver, J. Pohjanpelto, Differential invariant algebras of Lie pseudo-groups, Adv. Math. 222, no. 5, 1746-1792 (2009). 
[31] P. Olver, J. Pohjanpelto, F. Valiquette, On the structure of Lie pseudo-groups, SIGMA 5, 077 (2009).

[32] M. Rosenlicht, Some basic theorems on algebraic groups, Am. J. Math. 78, 401443 (1956).

[33] R. A. Sarkisyan, Rationality of the Poincaré series in Arnold's local problems of analysis, Izvestiya RAN Ser. Mat. (Izv.Math.) 74, no.2, 411-438 (2010).

[34] I. M. Singer, S. Sternberg, On the infinite groups of Lie and Cartan, J. d'Analyse Math. 15, 1-114 (1965).

[35] A.S. Shmelev, On differential invariants of some differential-geometric structures, Proc. Steklov Inst. Math. 209, 203-234 (1995).

[36] T. A. Springer, Invariant Theory, Lecture Notes in Math. 585, Springer-Verlag (1977).

[37] E. Stróżyna, H. Żolądek, The complete formal normal form for the BogdanovTakens singularity, Moscow Math. Jour. 15, no.1, 141-178 (2015).

[38] T.Y. Thomas, The Differential Invariants of Generalized Spaces, Cambridge University Press, Cambridge (1934).

[39] A. Tresse, Sur les invariants differentiels des groupes continus de transformations, Acta Math. 18, 1-88 (1894).

[40] A. Tresse, Détermination des invariants ponctuels de léquation différentielle ordinaire du second ordre $y^{\prime \prime}=\omega\left(x, y, y^{\prime}\right)$, Leipzig (1896).

[41] I. Vasiman, Symplectic Curvature Tensors, Mh. Math. 100, 299-327 (1985).

[42] S. M. Voronin, A. A. Grintchy, An analytic classification of saddle resonant singular points of holomorphic vector fields in the complex plane, Journ. Dynamical and Control Systems 2, no.I, 21-53 (1996).

[43] X. Wang, G. Chen, D. Wang, Unique normal forms for the Takens-Bogdanov singularity in a special case, C. R. Acad. Sci. Paris 332, Série I, 551-555 (2001).

[44] V. Yumaguzhin, Differential invariants of 2-order ODEs, Acta Applicandae Math. 109, no.1, 283-313 (2010).

[45] K. Zorawski, Über Biegungsinvarianten, Acta Math. 16, no.1, 1-64 (1892).

Department of Mathematics and Statistics, Uit the Arctic UniverSITY OF NorWAY, Troms $\varnothing$ 90-37, NorWAy. E-MAIL: boris.kruglikov@uit.no. 